Board of Governors of the Federal Reserve System

\author{
International Finance Discussion Papers
}

Number 526

October 1995

\title{
UNCERTAINTY, INSTRUMENT CHOICE, AND THE UNIQUENESS OF NASH EQUILIBRIUM: \\ MICROECONOMIC AND MACROECONOMIC EXAMPLES
}

Dale W. Henderson and Ning S. Zhu

NOTE: International Finance Discussion Papers are preliminary materials circulated to stimulate discussion and critical comment. References in publications to International Finance Discussion Papers (other than an acknowledgment that the writer has had access to unpublished material) should be cleared with the author or authors. 


\begin{abstract}
This paper contains two examples of static, symmetric, positive-sum games with two strategic players and a play by nature: (1) a microeconomic game between duopolists with joint costs facing uncertain demands for differentiated goods and (2) a macroeconomic game between two countries with inflation-bias preferences confronting uncertain demands for moneys. In both examples, each player can choose either of two variables as an instrument, and reaction functions are linear in the chosen instruments. With no uncertainty, there are four (Nash) equilibria, one for each possible instrument pair, because each player is indifferent between instruments given the instrument choice and instrument value of the other player. With uncertainty in the form of an additive disturbance, there are fewer equilibria because each player is not indifferent between instruments. These results are in accordance with the logic of Poole (1970) and Weitzman (1974) as explained by Klemperer and Meyer (1986) using examples of differentiated duopoly games with independent costs. In their main example with linear reaction functions, there is always a unique equilibrium. In contrast, in each of our examples with uncertainty, there is a unique equilibrium for some parameter values, but there are two equilibria for others. It is somewhat surprising that in both the Klemperer and Meyer example and our examples with unique equilibria, for some parameter values with the smallest amount of uncertainty the symmetric instrument pair chosen in the unique equilibrium is the one that yields the lower payoff with no uncertainty.
\end{abstract}




\section{UNCERTAINTY, INSTRUMENT CHOICE, \\ AND THE UNIQUENESS OF NASH EQUILIBRIUM: \\ MICROECONOMIC AND MACROECONOMIC EXAMPLES}

Dale W. Henderson and Ning S. Zhu ${ }^{1}$

\section{Introduction}

This paper contains two examples of static, symmetric, positive-sum games with two strategic players and a play by nature: (1) a microeconomic game between duopolists with joint costs facing uncertain demands for differentiated goods and (2) a macroeconomic game between two countries with inflation-bias preferences confronting uncertain demands for moneys. In both games, each player can choose either of two variables as an instrument. In our terminology, both are linear-reaction-function games because reaction functions are linear in the chosen instruments.

More than a century ago, it was discovered that there are both Cournot (1838) and Bertrand (1883) equilibria for duopoly games with no uncertainty. There are many examples of multiple (Nash) equilibria in linear-reaction-function games with no uncertainty. In the standard differentiated duopoly game with linear demands and independent, quadratic costs, there are four equilibria if each duopolist can choose either price or quantity as an instrumert. That is, there are as many equilibria as there are possible pairs of instrument

\footnotetext{
'We would like to thank Marc Dudey for suggesting the set up for the microeconomic example and Stephen Salant and Russell Cooper for helping us to clarify some concepts in game theory. Useful comments were provided by Harald Uhlig, our discussant, and others attending the conference entitled "Positive Political Economy: Theory and Evidence" at the CentER for Economic Research of Tilburg University: by Jon Faust; and by David Bowman, Andrew Levin, and others attending a seminar at the Board of Governors of the Federal Reserve System. The paper will appear in Eijffinger and Huizinga (1996). It reflects the views of the authors and should not be interpreted as reflecting e ther the views of the Board of Governors of the Federal Reserve System or other members of its staff, or the views of the World Bank, its Executive Board of Directors, its member countries, or other members of its staff.
} 
choices. Likewise, in two-player macroeconomic games with quadratic utilities ard linear economies there are as many equilibria as there are possible pairs of instrument choices. ${ }^{2}$

The explanation of the existence of multiple equilibria in linear-reaction-function games with no uncertainty is the same as the explanation of a familiar result. Poole (1970) and Weitzman (1974) show that with no uncertainty a single controller is indifferent among instruments. Likewise, with no uncertainty if one player chooses his instrument and sets a value for it, the other is indifferent among instruments. It follows that, for example, if each of two players can choose one of two variables as an instrument, there are four equilibria. ${ }^{3}$

Klemperer and Meyer (1986) modify differentiated duopoly games by introducing uncertainty in the form of a play by nature that is unknown to the duopolists. Introducing uncertainty in this form is of interest in and of itself. However, Klemperer and Meyer find that it results in a game with different qualitative properties: the number of equilibria is smaller than with no uncertainty.

In their main example, Klemperer and Meyer modify the standard differentiated duopoly game by assuming that the demands have a common additive disturbance. This example has the very attractive property that there is a unique equilibrium for every set of parameter values. It also has a somewhat surprising property not emphasized by Kilemperer and Meyer. For some parameter values, the symmetric instrument pair chosen in the unique

\footnotetext{
'Giavazzi and Giovannini (1989) and Canzoneri and Henderson (1988) compare an equilibrium in which each of two coun ries choose money supplies as instruments with an equilibrium in which one chooses the money supply and the other chooses the exchang: rate. Turnovsky and d'Orey (1986), Canzoneri and Henderson (1989), and Henderson and Zhu (1990) compare the four equilibria which are possible when each of two countries can choose either the money supply or the interest rate as its instrument. Tabellini (1987) compares the two equilibria which are possible when the monetary authority can choose either the money supply or the interest rate as its in strument and the fiscal authority chooses government expenditures as its instrument.

'In linear-reaction-function games, there can be multiple equilibria only if there are two or more possible pairs of instrument choices. However, in nonlinear-reaction-function games, there can be multiple equilibria even if there is only one possible pair of instnıment choices as explained in. for example, Cooper and John (1988).
} 
equilibrium is the one that yields lower profits with no uncertainty.

The explanation of the reduction in the number of equilibria with uncertainty is the same as the explanation of another familiar result. Poole (1970) and Weitzman (1974) show that with uncertainty a single controller is not indifferent among instruments. Likewise, with uncertainty if one player chooses his instrument and sets a value for it, the other is not indifferent among instruments. Since players are not indifferent among instruments, the number of equilibria is reduced, perhaps to one.

The two examples in this paper are designed to shed further light on the implications of introdıcing uncertainty in the form of a play by nature into linear-reaction-function games for the number of equilibria and the characteristics of these equilibria. The microeconomic example is an extension of the main Klemperer and Meyer (1986) example to the case of joint costs. The macroeconomic example is a two-country version of the Kydland-Prescott (1977) and Barro-Gordon (1983) inflation-bias game with uncertain demands for money similar to the one analyzed by Henderson and Zhu (1990). In the macroeconomic example, each country can choose either the money supply or the interest rate as its instrument.

Our examples yield results similar to those of Klemperer and Meyer in some respects. With no uncertainty there are four equilibria, one for each possible instrument pair. Introducing uncertainty results in a game with fewer equilibria. In some cases in which the equilibriım is unique, the smallest amount of uncertainty causes the players to choose the symmetric instrument pair that is worse for them with no uncertainty. However, our example:; yield results different from those of Klemperer and Meyer in an important respect. For some parameter values, introducing uncertainty results in a game with two equilibria. 


\section{A Microeconomic Example: Differentiated Duopoly with Uncertain Demands}

In the microeconomic example, we use a model of duopoly with differentiated goods.

First, we lay out a model with linear demands; joint, quadratic costs; and an additive disturbance that affects both demands. Then, we discuss the multiplicity of equilibria with no uncertainty. Finally, we consider the implications of introducing uncertainty in the form of the additive demand disturbance. We analyze both the special case of independent costs for which Klemperer and Meyer (1986) obtain their uniqueness result and the general case of joint costs.

\section{A. The Model}

In our model, the symmetric duopolists, nohat and hat, each produce and sell a single differentiated good. Variables with no hats and hats over them are possible instrument variables for nohat and hat, respectively. Prices received $(P$ and $\hat{P})$ are related to quantities sold ( $Q$ and $\hat{Q}$ ) by the inverse demand functions

$$
P=a-.5 b Q-f \hat{Q}+\eta, \quad \hat{P}=a-.5 b \hat{Q}-f Q+\eta,
$$

where $\eta$ is a disturbance with zero mean and finite variance. The price of each gcod falls with the quantity of that good sold $(b>0)$. We assume that the two goods are substitutes, so the price of each good also falls with the quantity of the other good sold $(f>0){ }^{4}$ We also assume that the price of a good falls if the quantity of that good sold increases and the quantity of the other good sold decreases by the same amount $(b>2 f)$.

\footnotetext{
${ }^{4}$ The case in which the two goods are complements so that the price of each good rises with the quantity of the other good : :old $(f<0)$ is considered in Henderson and Zhu (forthcoming).
} 
We assume that the total costs of the duopolists ( $C$ and $\hat{C}$ ) are the following quadratic functions of their outputs,

$$
C=g Q+.5 k Q^{2}+u Q \hat{Q}, \quad \hat{C}=g \hat{Q}+.5 k \hat{Q}^{2}+u \hat{Q} Q
$$

so the duopolists have joint costs. ${ }^{5}$ We assume that $0 \leq g<a$, but we consider both positive and negative values for $k$ and $u$.

$\mathrm{W}$ e assume that the duopolists are involved in a static, positive-sum game with simultant:ous play in which nature also makes a play. Each duopolist has two possible instruments, quantity and price. Each must chose an instrument and the value at which to set that instrument before uncertainty is resolved. Since the duopolists are symmetric, we need consider in detail the behavior of only one of them, say nohat.

Nohat's profits when nohat chooses instrument $j$ and hat chooses instrument $\hat{j}$ are represented by $\Pi^{\hat{j}}(j, \hat{j} ; \eta)$. Nohat's profits for the four possible pairs of instrument choices are given by equations (T1.1) through (T1.4) in Table 1. Nohat's zero-disturbance profits for values of an instrument pair $j$ and $\hat{j}$ are defined as what nohat's profits would be for values of: that instrument pair if the disturbance term were zero and are represented by $\Pi^{\hat{j}}(j, \hat{j} ; 0)$. Our assumptions about the parameters of the demand and cost functions imply

${ }^{5}$ These cost functions are special cases of the general quadratic cost functions

$$
C=g Q+h \hat{Q}+.5 k Q^{2}+u Q \hat{Q}+.5 x \hat{Q}^{2}, \quad \hat{C}=g \hat{Q}+h Q+.5 k \hat{Q}^{2}+u \hat{Q} Q+.5 x Q^{2} .
$$

With $x \neq 0$, the analysis is only a little more complicated, but with $h \neq 0$, the analysis is considerably more complicated. Yohe (1979) uses models with general quadratic cost functions like those in equations (F5.1). 
that nohat's zero disturbance profits for the instrument pair $j$ and $\hat{j}$ are a concave function of nohat's instrument $j$ for any given value of hat's instrument $\hat{j}^{6}$

\section{B. The Model with No Uncertainty}

\section{The Multiplicity of Equilibria}

With no uncertainty $\left(\sigma_{\eta}^{2}=0\right)$, we obtain the familiar result that there are four equilibria no matter whether costs are independent $(u=0)$ or joint $(u \neq 0)$. For any given value of a particular instrument variable chosen by hat, the maximum value of nohat's zerodisturbance profits is the same no matter whether nohat chooses $Q$ or $P$ as an instrument because there is a one to one relationship between $Q$ and $P:{ }^{7}$

$$
\max _{Q}\left[\Pi^{Q \hat{j}}(Q, \hat{j} ; 0)\right]=\max _{P}\left[\Pi^{P \hat{j}}(P, \hat{j} ; 0)\right]
$$

This logic is in accordance with the logic used by Poole (1970) and Weitzman (1974) to

${ }^{6}$ That is,

$$
\begin{aligned}
& \Pi^{P \hat{Q}}(P, \hat{Q} ; \eta)=\Pi^{Q \dot{Q}}\left[Q^{P \hat{Q}}(P, \hat{Q} ; \eta), \hat{Q} ; \eta\right], \quad \Pi^{Q \hat{P}}(Q, \hat{P} ; \eta)=\Pi^{Q Q}\left[Q, \hat{Q}^{Q \dot{P}}(Q, \hat{P} ; \eta) ; \eta\right], \\
& \Pi^{P \hat{P}}(P, \hat{P} ; \eta)=\Pi^{Q \hat{\phi}}\left[Q^{P \hat{P}}(P, \hat{P} ; \eta), \hat{Q}^{P \hat{P}}(P, \hat{P} ; \eta) ; \eta\right] \text {, }
\end{aligned}
$$

where $Q^{P Q}(\cdot)$ is obtained by solving the first equation in equations (1) for $Q, \hat{Q}^{Q \hat{P}}(\cdot)$ is obtained by solving the second equition in equations (1) for $\hat{Q}$, and $Q^{P \hat{P}}(\cdot)$ and $\hat{Q}^{P \hat{P}}(\cdot)$ are obtained by solving equations (1) simultaneously for $Q$ and $\hat{Q}$. The expressions for $Q^{P \hat{Q}}, \hat{Q} \hat{Q}^{Q \dot{P}}, Q^{P \hat{P}}$, and $\hat{Q}^{P \hat{P}}$ are

$$
\begin{aligned}
Q^{P \hat{Q}}(P, \hat{Q} ; \eta) & =\left(\frac{2}{b}\right)(a-P-f \hat{Q}+\eta), \quad \hat{Q}^{Q \dot{P}}(Q, \hat{P} ; \eta)=\left(\frac{2}{b}\right)(a-\hat{P}-f Q+\eta), \\
Q^{P \hat{P}}(P, \hat{P} ; \eta) & =\left(\frac{2}{\Delta}\right)[(b-2 f) a-b P+2 f \hat{P}+(b-2 f) \eta], \\
\hat{Q}^{P \dot{P}}(P, \hat{P} ; \eta) & =\left(\frac{2}{\Delta}\right)[(b-2 f) a-b \hat{P}+2 f P+(b-2 f) \eta], \\
\Delta & =b^{2}-4 f^{2} .
\end{aligned}
$$

\footnotetext{
'Of course. if nohat could use his instrument choice to affect hat's instrument choice, he would no longer be indifferent be ween instruments.
} 
establish that with no uncertainty a single controller is indifferent among instruments.

It follows that there are four equilibria in which the instrument pairs are $Q$ and $\hat{Q}, P$ and $\hat{Q}, Q$ and $\hat{P}$, and $P$ and $\hat{P}$, respectively. We refer to these equilibria as the $Q \hat{Q}, P \hat{Q}$, $Q \hat{P}$, and $P \hat{P}$ equilibria, respectively. As an example, wo establish that there is a $P \hat{Q}$ equilibrium. Begin with nohat's and hat's profits functions when $P$ and $\hat{Q}$ are chosen as instruments. Set both the first derivative of nohat's profit function ith respect to $P$ and the first derivative of hat's profit function with respect to $\hat{Q}$ equal to zero. Thi. ir of first order conditions is a pair of simultaneous linear equations in $P$ and $\hat{Q}$. It remains to demonstrate that the solution to this pair of first order conditions is an equilibrium. Given the soluticn for $\hat{Q}$, the solution for $P$ is the best response for nohat given that he chooses $P$ as an instrument. Likewise, given the solution for $P$, the solution for $\hat{Q}$ is the best response for hat given that he chooses $\hat{Q}$ as an instrument. As we argue in the preceding paragraph, nohat can do no better by choosing $Q$ as an instrument. Likewise, hat can do no better by choosing $\hat{P}$ as an instrument. Therefore, the solution to the pair of first order conditions when $P$ and $\hat{Q}$ are chosen as instruments is an equilibrium. In a similar manner, it can be established that there are $Q \hat{Q}, Q \hat{P}$, and $P \hat{P}$ equilibria.

There is a certain fragility to the set of equilibria. Consider the $P \hat{Q}$ equilibrium. With no uncertainty, nohat is indifferent between using $P$ as an instrument and using $Q$. Of 
course, if there is to be a $P Q \hat{Q}$ equilibrium, he must actually use $P$. If the game structure

were altered so that nohat had even a slight preference for $Q$, then there would be no $P \hat{Q}$

equilibrium. As we confirm below, with uncertainty nohat is not indifferent between

instruments.

\section{The Comparison of Profits at Three Points}

In this subsection we compare profits at the symmetric efficient point with those in the $Q \hat{Q}$ (Cournot) and $P \hat{P}$ (Bertrand) equilibria for both the case of independent costs $(u=0)$

and the case of joint costs $(u \neq 0)$. We use Figures 1 and 2 , which are diagrams in $Q \hat{Q}$

space. $^{8}$ The comparison is of interest in and of itself, but our main reason for making it is so that we can refer to it in the analysis of the uncertainty case.

The Efficient Point. As a standard of comparison, we use the symmetric efficient point, the point at which the simple sum of the profits of the duopolists is maximized. We refer to this point as "the" efficient point for short and represent it by point $\mathrm{E}$ in Figures 1 and 2. Since the duopolists are symmetric, they produce equal quantities at this point. At the efficient point, it must be impossible to make either duopolist better cff without making the other worse off, and the quantities produced must be equal. Therefore, at $\mathrm{E}$ an isoprofit locus for nohat must be tangent to an isoprofit locus for hat on the $45^{\circ}$ line:

\footnotetext{
${ }^{k}$ Throughout this footnote we refer to results for symmetric duopolists whose products are substitutes (f $>0$ ) or complements (f $<0$ ). Singh and Vives (1984), Vives (1985), and Cheng (1985) analyze the case of constant marginal costs. Singh and Vives and Vives establish algebraically and Cheng establishes graphically that profits are higher or lower in the $Q \hat{Q}$ equilibrium than in the $P \hat{P}$ equilibriun depending on whether the products are substitutes or complements. Xiang (1993) analyzes the case of independent variable marginal costs. In Henderson and Zhu (forthconing) we generalize the analyses of these authors to the case of joint costs. In the text of the current paper we apply a slightly modified version of Cheng's graphical technique to derive results for substitutes with joint costs. The slight modification of Cheng's technique is that we operate in $Q \hat{Q}$ space rather than in $P \hat{P}$ space as Cheng did.
} 


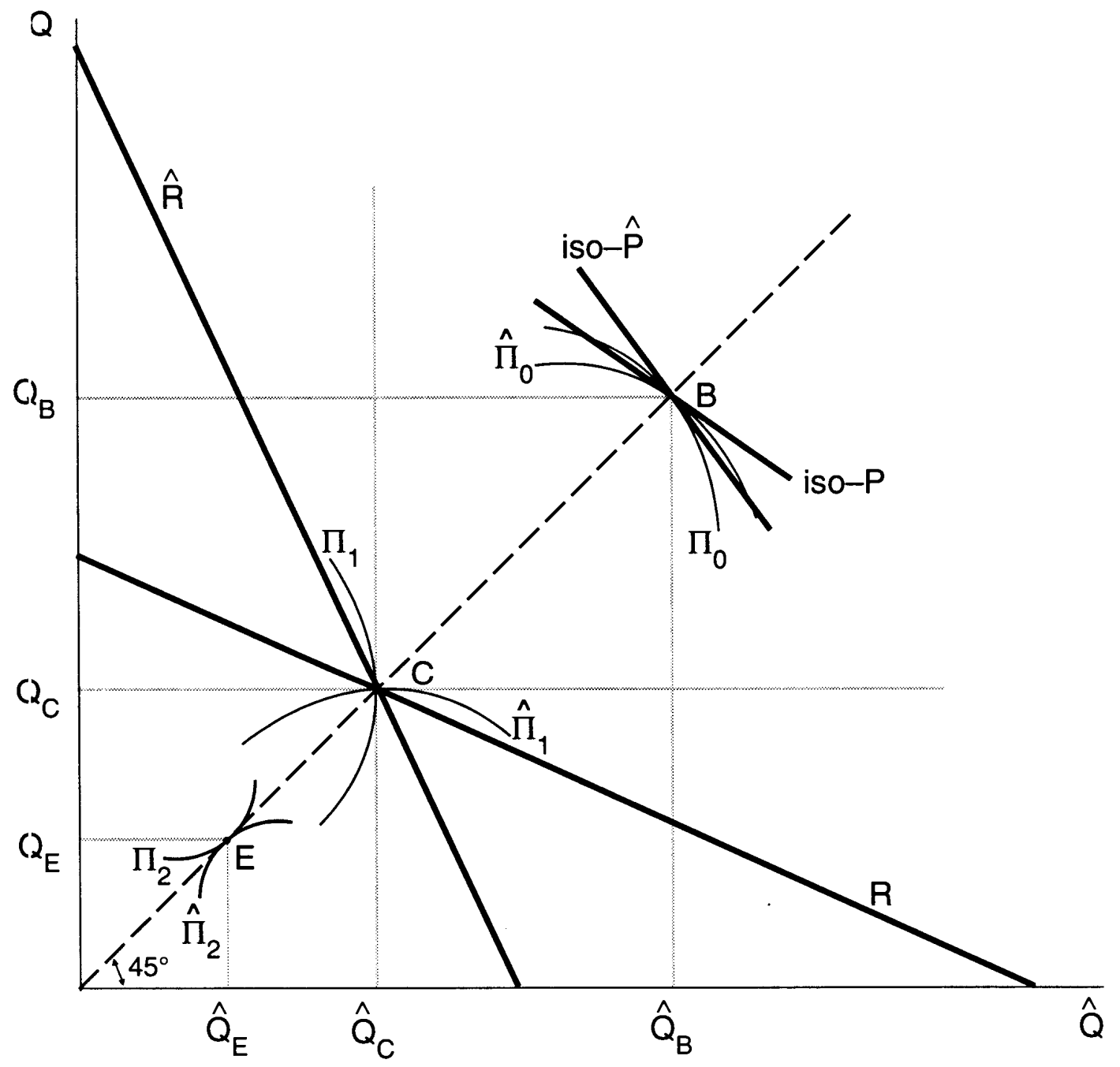

Figure 1 


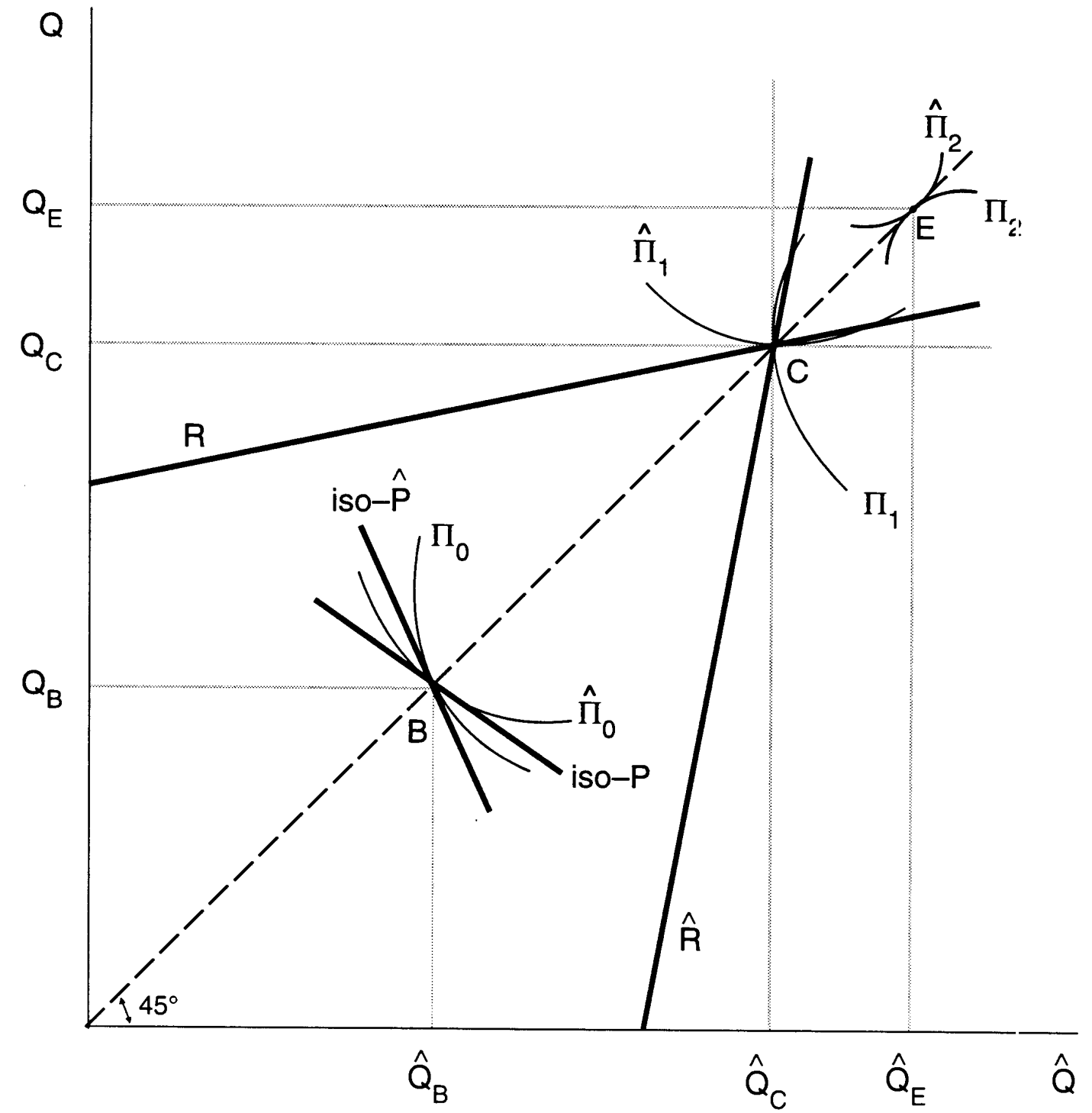

Figure 2 


$$
\left[\frac{d Q}{d \hat{Q}}\right]_{d \Pi=0, Q=\hat{Q}}=\left[\frac{d Q}{d \hat{Q}}\right]_{d \hat{\Pi}=0, Q=\hat{Q}}
$$

Since the duopolists are symmetric, at any point on the $45^{\circ}$ line the slopes of their isoprofit loci are reciprocals. This fact and equation (4) imply that

$$
\left[\frac{d Q}{d \hat{Q}}\right]_{d \Pi=0, Q=\hat{Q}}=1
$$

The slope: of nohat's isoprofit locus is

$$
\left[\frac{d Q}{d \hat{Q}}\right]_{d \Pi=0}=-\frac{\Pi_{\hat{Q}}^{Q \hat{Q}}}{\Pi_{Q}^{Q \hat{Q}}}=-\frac{-(f+u) Q}{a-g-(b+k) Q-(f+u) \hat{Q}}
$$

Therefore, the condition in equation (5) becomes ${ }^{9}$

$$
\left[\frac{d Q}{d \hat{Q}}\right]_{d \Pi=0, Q=\hat{Q}}=\frac{(f+u) Q}{a-g-(b+k+f+u) Q}=1
$$

Solving for $Q$ yields the quantities produced at the efficient point, $Q^{E}=\hat{Q}^{E}$ :

${ }^{y}$ In algebraic terms, necessary and sufficient conditions for being at the efficient point are obtained by setting $\hat{Q}$ equal to $Q$ in the expression fo: nohat's profits in equation (T1.1) and differentiating. The first derivative of the resulting expression must be equal to zero,

$$
\frac{d\left[\left(\Pi^{Q Q}\right)_{Q=\hat{Q}}\right]}{d Q}=a-g-[b+k+2(f+u)] Q=0,
$$

as it is if the condition in equation (7) is met. The second derivative must be negative,

$$
\frac{d^{2}\left[\left(\Pi^{Q Q}\right)_{Q=\dot{Q}}\right]}{d Q^{2}}=-[b+k+2(f+u)]<0,
$$

as it is if the parameters of the model are chosen appropriately. Note that equation (F9.2) implies that $b+k+2(f+u)>0$, so equation (F9.1) implies that $Q^{E}=\hat{Q}^{E}>0$ if and only if $a-g>0$. 


$$
Q^{E}=\hat{Q}^{E}=\frac{a-g}{b+k+2(f+u)}
$$

The $Q \hat{Q}$ Equilibrium. In the $Q \hat{Q}$ equilibrium, nohat maximizes profits taking $\hat{Q}$ as given. ${ }^{10}$ Given values of $\hat{Q}$ are represented by vertical lines in Figures 1 and 2 . When the duopolists choose symmetric instruments, the quantities they produce must be the same. Therefore, in the $Q \hat{Q}$ equilibrium an isoprofit locus for nohat must be tangent to a vertical line at a point on the $45^{\circ}$ line as it is at points $\mathrm{C}$ (for Cournot) in Figures 1 and 2 . This condition is met if and only if

$$
\left[\frac{d Q}{d \hat{Q}}\right]_{d I I=0, Q=\hat{Q}}=\frac{(f+u) Q}{a-g-(b+k+f+u) Q} \rightarrow \pm \infty
$$

$f+u$ is always positive when $u$ is zero or positive, and we assume that $f+u \neq 0$ if $u$ is negative. It follows that the condition in equation (9) implies that

\footnotetext{
"In algebraic terms, necessary and sufficient conditions for being at the $Q \hat{Q}$ equilibrium are obtained by differentiating the expression for nohat's profits in equation (T1.1) with respect to $Q$ and setting $\hat{Q}$ equal to $Q$ in the resulting expressions. The first order zondition is that

$$
\left[\Pi_{Q}^{Q Q}\right]_{Q=\dot{Q}}=a-g-(b+k+u) Q=0,
$$

as it is when the condition in equation (10) is met. The second order condition is that

$$
\Pi_{Q Q}^{Q Q}=-(b+k)<0,
$$

which implies that $b+k=0$. Since we must have $b+k+2(f+u)>0$ in order for the efficient point to be a constrained maxinum, $b+k+f+u>0$. Since we must have $a-g=0$ in order for outputs to be positive at the efficient point, equation (F10.1) inmlies that $Q^{C}=\hat{Q}^{C}>0$
} 


$$
a-g-(b+k+f+u) Q=0
$$

Solving for $Q$ yields the quantities sold in the $Q \hat{Q}$ equilibrium, $Q^{c}=\hat{Q}^{c}$ :

$$
Q^{c}=\hat{Q}^{c}=\frac{a-g}{b+k+f+u} \text {. }
$$

The $P \hat{P}$ Equilibrium. In the $P \hat{P}$ equilibrium, nohat maximizes profits taking $\hat{P}$ as given. ${ }^{11}$ Values of $\hat{P}$ are represented by downward-sloping iso- $\hat{P}$ lines in Figures 1 and 2 .

Solving the second equation in equations (1) for $Q$ yields the equation for iso- $\hat{P}$ lines:

$$
f Q=a-\hat{P}-.5 b \hat{Q}
$$

which has the slope $-\frac{b}{2 f}<-1$ relative to the $\hat{Q}$ axis. When the duopolists choose symmetric: instruments, the quantities they produce must be the same. Therefore, in the $P \hat{P}$ equilibrium an isoprofit locus for nohat must be tangent to an iso- $\hat{P}$ line at a point on the $45^{\circ}$

\footnotetext{
"In algebr.ic terms, the version of the necessary and sufficient conditions for being at the $\boldsymbol{P} \hat{\boldsymbol{P}}$ equilibrium that is most useful for present purposes can $b \geq$ derived by differentiating the expression for nohat's profits as a function of $Q$ and $\hat{Q}$ in equation (T1.1) with respect to $P$ using the chain rule. The first order condition is that$$
\left[\Pi_{P}^{P \dot{P}}\right]_{Q=Q}=\frac{2 b}{\Delta}\left\{a-g-\left[b+k+\left(1-\frac{2 f}{b}\right)(f+u)\right] Q\right\}=0,
$$

as it is when the condition equation (13) is met. The second order condition is that

$$
\Pi_{P P}^{P \dot{P}}=-(b+k)\left(\frac{4 b^{2}}{\Delta^{2}}\right)-(f+u)\left(\frac{16 b f}{\Delta^{2}}\right)=-\left(\frac{4 b}{\Delta^{2}}\right)[b(b+k)-4 f(f+u)]<0,
$$$$
\Delta=b^{2}-4 f^{2} \text {, }
$$

which implies that $b(b+k)-4 f(f+u)>0$. Since we must have $b+k+2(f+u)>0$ in order for the efficient point to be a constrained maximum, $b+k+\left(1-\frac{2 f}{b}\right)(f+u)>0$. Since we nust have $a-g>0$ in order for outputs to be positive at the efficient point. equation (F11.1) implies that $Q^{B}=\hat{Q}^{B}>0$.
} 
line as it is as points B (for Bertrand) in Figures 1 and 2. This condition is met if and only if

$$
\left[\frac{d Q}{d \hat{Q}}\right]_{d \Pi=0, Q=\hat{Q}}=\frac{(f+u) Q}{a-g-(b+k+f+u) Q}=-\frac{b}{2 f}
$$

Solving for $Q$ yields the quantities produced in the $P \hat{P}$ equilibrium, $Q^{B}=\hat{Q}^{B}$ :

$$
Q^{B}=\hat{Q}^{B}=\frac{a-g}{b+k+\left[1-\frac{2 f}{b}\right](f+u)} .
$$

The Reaction Functions and the Isoprofit Loci. The reaction function fos: nohat represented by $R$ in Figures 1 and 2 gives the optimal $Q$ for each value of $\hat{Q}$ when $Q$ and $\hat{Q}$ are the instruments. The equation for $R$ is obtained by setting $\Pi_{Q}^{Q Q}$, which is given by the denominator of the last term in equation (6), equal to zero and solving for $Q$ to obtain

$$
(b+k) Q=(a-g)-(f+u) \hat{Q}
$$

$R$ has a positive intercept but may have a negative or positive slope as in Figures $I$ and 2 , respectively, depending on whether $f+u \gtrless 0$.

At their intersections with $R$, iso-profit loci for nohat have slopes approaching plus or minus infinity with respect to the $\hat{Q}$ axis and slopes of zero with respect to the $Q$ axis: 


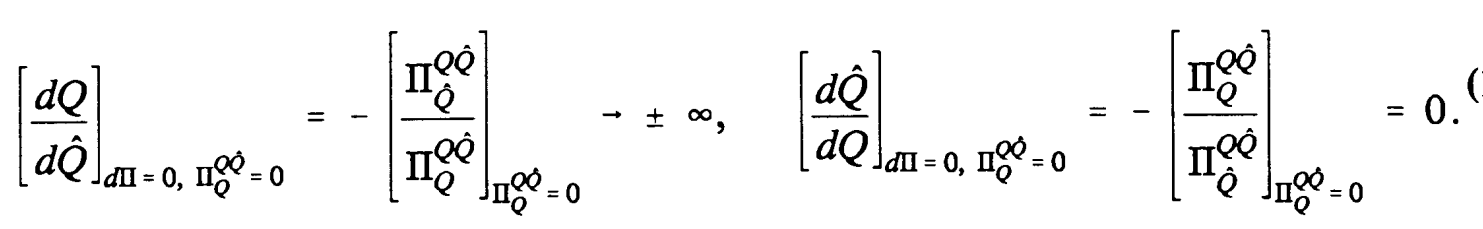

Expressions for $\Pi_{Q}^{Q \hat{Q}}$ and $\Pi_{\hat{Q}}^{Q \hat{Q}}$ are given in equation (6). $\Pi_{\hat{Q}}^{Q \hat{Q}} \neq 0$ by assumption, and $\Pi_{Q}^{Q \hat{Q}}=0$ on $R$. At their intersections with $R$, isoprofit loci for nohat are concave or convex to the $Q$ axis as in Figures 1 and 2, respectively, depending on whether $f+u \gtrless 0::^{12}$

$$
\left[\frac{d\left[\left(\frac{d \hat{Q}}{d Q}\right]_{d \Pi=0}\right)}{d Q}\right]_{\Pi_{Q}^{Q Q}=0}=-\frac{\Pi_{Q Q}^{Q \hat{Q}}}{\Pi_{\hat{Q}}^{Q \hat{Q}}}=-\frac{b+k}{f+u} .
$$

Thus, at their intersections with $R$, isoprofit loci for nohat are concave to the $Q$ axis if $R$ is downwarc. sloping and are convex to the $Q$ axis if $R$ is upward sloping.

The $P \hat{P}$ equilibrium is the point on the $45^{\circ}$ line at which an isoprofit locus for nohat is tangent to an iso- $\hat{P}$ line and an isoprofit locus for hat is tangent to an iso- $P$ line. The $P \hat{P}$ equilibrium must lie to the northeast or southwest of the $Q \hat{Q}$ equilibrium as in Figures 1 and

"The change in the slope of an isoprofit locus with respect to the Q axis is

$$
\frac{d\left[\left[\frac{d \hat{Q}}{d Q}\right]_{d \Pi=0}\right)}{d Q}=-\left[\frac{1}{\Pi_{\dot{Q}}^{Q \dot{Q}}}\right]^{3}\left[\Pi_{Q \dot{Q}}^{Q \dot{Q}}\left(\Pi_{\dot{Q}}^{Q \dot{Q}}\right)^{2}-2 \Pi_{Q \dot{Q}}^{Q \dot{Q}} \Pi_{Q}^{Q \dot{Q}} \Pi_{\dot{Q}}^{Q \dot{Q}}+\Pi_{\dot{Q} \dot{Q}}^{Q \dot{Q}}\left(\Pi_{Q}^{Q \dot{Q}}\right)^{2}\right]
$$

and the result $\mathrm{n}$ equation (17) follows because $\Pi_{Q}^{Q \dot{Q}}=0$ on $R$. 
2 , respectively, depending on whether $f+u \gtrless 0$.

The Comparison. The ranking of the quantities produced in the $Q \hat{Q}$ and $P \hat{P}$ equilibria and at the efficient point depends on the sign of $f+u$, but the ranking of profits does not. ${ }^{13}$ It follows from the graphical analysis above that if $f+u>0$, then the $P \hat{P}$ equilibrium lies to the northeast of the $Q \hat{Q}$ equilibrium which lies to the northeast of the efficient point as in Figure 1 so that

$$
Q^{E}=\hat{Q}^{E}<Q^{C}=\hat{Q}^{C}<Q^{B}=\hat{Q}^{B},
$$

but that if $f+u<0$, then the $P \hat{P}$ equilibrium lies to the southwest of the $Q \hat{Q}$ equilibrium which lies to the southwest of the efficient point as in Figure 2, so that

$$
Q^{B}=\hat{Q}^{B}<Q^{C}=\hat{Q}^{C}<Q^{E}=\hat{Q}^{E} .
$$

These rankings follow from equations (8), (11), and (14) and our assumption that $b>2 f$.

$Q$ and $\hat{Q}$ are smaller or larger at the efficient point than at the $Q \hat{Q}$ equilibrium and the $P \hat{P}$ equilibrium depending on whether the spillover effects of quantity increases are negative or positive and depending on whether the spillover effects of price increases are positive or negative, respectively. One duopolist's instrument is said to have a positive spillover effect or a negative spillover effect depending on whether an increase in that

\footnotetext{
${ }^{13}$ Recall that we assume throughout that $f>0$. Our result that the ranking of profits in the $Q \hat{Q}$ and $P \hat{P}$ equilibria is indejendent of the sign of $f+u$ given that $f>0$ does not conflict with the standard result that the ranking of profits in the $Q \hat{Q}$ and $P \hat{P}$ equilibria with independent costs depends on the sign of $f$ as shown for example in Singh and Vives (1984).
} 
instrument raises or lowers the other duopolist's profits. ${ }^{14}-(f+u) \hat{Q}$ and $-(f+u) Q$ are the spillover effects of $Q$ on $\hat{\Pi}$ and $\hat{Q}$ on $\Pi$, respectively, and $\left[1-\frac{2 f}{b}\right](f+u) \hat{Q}$ and $\left[1-\frac{2 f}{b}\right](f+u) Q$ are the spillover effects of $P$ on $\hat{\Pi}$ and $\hat{P}$ on $\Pi$, respectively. $Q$ and $\hat{Q}$ are positive, so the spillover effects of $Q$ and $\hat{Q}$ are negative or positive and the spillover effects of $P$ and $\hat{P}$ are positive or negative depending on whether $f+u \gtrless 0$. If the duopolists cooperate, they take account of spillover effects and attain the efficient point. However, if they behave noncooperatively, they do not take these effects into account. For example, if $f+u>0$ so that the spillover effects of $Q$ and $\hat{Q}$ are negative and the spillover effects of $P$ and $\hat{P}$ are positive, nohat does not take into account the fact that an increase in his quantity lowers hat's profits or the fact that an increase in his price raises hat's profits. As a result, he chooses a larger quantity or a lower price than he would at the efficient point. Of course, if he is to obtain a lower price he must sell a larger quantity.

$Q$ and $\hat{Q}$ are larger or smaller in the $P \hat{P}$ equilibrium than in the $Q \hat{Q}$ equilibrium depending on whether the quantities of the two goods are strategic substitutes or strategic complements. The quantities of the two goods are said to be strategic substitutes or strategic complements depending on whether an increase in one quantity lowers or raises the marginal profit of ircreasing the other quantity, that is, depending on whether $\Pi_{Q \dot{Q}}^{Q \hat{Q}}=f+u \gtrless 0$. If hat chooses $\hat{P}$ instead of $\hat{Q}$ as an instrument, then when nohat increases $Q$, hat must reduce $\hat{Q}$ in order to keep $\hat{P}$ from falling. If the two quantities are strategic substitutes, a decrease

\footnotetext{
${ }^{14}$ For a concise statement of the standard definitions of positive spillover effect, negative spillover effect, strategic substitutes, and strategic comple nents see Cooper and John (1988).
} 
in $\hat{Q}$ raises nohat's marginal profit, so the value of $Q$ at which nohat's marginal profit is zero is larger. For analogous reasons, if nohat chooses $P$ instead of $Q$ as an instrument, the value of $\hat{Q}$ at which hat's marginal profit is zero is larger. Therefore, $Q$ and $\hat{Q}$ are larger in the $P \hat{P}$ equilibrium than in the $Q \hat{Q}$ equilibrium.

Profits at the efficient point are always higher than profits in the QQ equilibrium which are always higher than profits in the $P \hat{P}$ equilibrium:

$$
\Pi^{B}=\hat{\Pi}^{B}<\Pi^{C}=\hat{\Pi}^{C}<\Pi^{E}=\hat{\Pi}^{E} .
$$

Profits increase, remain unchanged, or decrease with increases in $Q=\hat{Q}$ depending on whether $Q=\hat{Q} \lesseqgtr Q^{E}=\hat{Q}^{E} \cdot{ }^{15}$ The $P \hat{P}$ equilibrium is always farther from the efficient point than the $Q \hat{Q}$ equilibrium along the $45^{\circ}$ line.

\section{The Implications of Introducing an Additive Demand Disturbance}

\section{Preliminaries}

In this section we consider the implications of introducing uncertainty into our differentiated duopoly game with joint costs in the form of an additive disturbance term that affects both demand functions $\left(\sigma_{\eta}^{2}>0\right)$. Klemperer and Meyer (1986) discovered that with uncertainty of this form there is only one equilibrium in a differentiated duopoly game with independent costs. They obtain their result because with this kind of uncertainty nohat is not

\footnotetext{
${ }^{\text {Is When }} \hat{Q}$ is kept equal to $Q$ and the conditions in footnote 9 are met, nohat's profit function is concave in $Q$ and has a unique maximum at $Q^{E}=\hat{Q}^{E}$.
} 
indifferent between choosing $Q$ or $P$ as an instrument for a given value of hat's instrument. The logic they use to establish this result is in accordance with the logic used by Poole (1970) and Weitzman (1974) to establish that with uncertainty a single controller is not indifferent among ins:ruments. There is an important similarity between the case of independent costs considered by Klemperer and Meyer and the case of joint costs considered here: the number of equilibria is smaller with uncertainty than without it. However, there is also an important difference between the two cases: with independent costs, there is only one equilibrium for all parameter values, but with joint costs, whether there is one equilibrium or two depends on parameter values.

Recall that we assume that demands are linear, that costs are quadratic, and that the only kind of uncertainty is an additive disturbance that enters both demand functions. These assumptiors have the familiar implication that nohat's expected profits for values of an instrument pair $j$ and $\hat{j}$ can be written as the sum of nohat's zero-disturbance profits for those value:s of that instrument pair and a linear function of the variance of the disturbance:

$$
E \Pi^{\hat{j}}(j, \hat{j} ; \eta)=\Pi^{\hat{j}}(j, \hat{j} ; 0)+\ell^{\hat{j}}\left(\sigma_{\eta}^{2}\right)
$$

It is important to note that the linear function $\ell^{\hat{j}}\left(\sigma_{\eta}^{2}\right)$ depends only on which instrument pair is chosen and not on the values at which the instruments are set.

Consequently, the maximized value of nohat's expected profits for instrument $j$ given a value of instrument $\hat{j}$ can be written as the sum of the maximized value of nohat's zerodisturbance: profits for instrument $j$ given that value of instrument $\hat{j}$ and the same linear 
function of the variance of the disturbance:

$$
\max _{j}\left[E \Pi^{\hat{j}}(j, \hat{j} ; \eta)\right]=\max _{j}\left[\Pi^{\hat{j}}(j, \hat{j} ; 0)\right]+\ell^{\hat{j}}\left(\sigma_{\eta}^{2}\right)
$$

where $\max _{j}\left[E \Pi^{\hat{j}}(j, \hat{j} ; \eta)\right]$ represents the maximized value of nohat's expected profits for the instrument $j$ given a value for the instrument $\hat{j}$, and $\max _{j}[\Pi \hat{j}(j, \hat{j} ; 0)]$ represents the maximized value of nohat's zero-disturbance profits for the instrument $j$ given a value for the instrument $\hat{j}$.

Evaluating $\ell^{Q \hat{Q}}\left(\sigma_{\eta}^{2}\right), \ell^{P \hat{Q}}\left(\sigma_{\eta}^{2}\right), \ell^{Q \hat{P}}\left(\sigma_{\eta}^{2}\right)$, and $\ell^{P \hat{P}}\left(\sigma_{\eta}^{2}\right)$ using equations (T1.1): (T1.2), (T1.3), and (T1.4) and inserting them into the appropriate version of equation (22) yield

$$
\begin{aligned}
& \max _{Q}\left[E \Pi^{Q \hat{Q}}(Q, \hat{Q} ; \eta)\right]=\max _{Q}\left[\Pi^{Q \hat{Q}}(Q, \hat{Q} ; 0)\right], \\
& \max _{P}\left[E \Pi^{P \hat{Q}}(P, \hat{Q} ; \eta)\right]=\max _{P}\left[\Pi^{P \hat{Q}}(P, \hat{Q} ; 0)\right]-2 k\left(\frac{1}{b}\right)^{2} \sigma_{\eta}^{2}, \\
& \max _{Q}\left[E \Pi^{Q \hat{P}}(Q, \hat{P} ; \eta)\right]=\max _{Q}\left[\Pi^{Q \hat{P}}(Q, \hat{P} ; 0)\right], \\
& \max _{P}\left[E \Pi^{P \hat{P}}(P, \hat{P} ; \eta)\right]=\max _{P}\left[\Pi^{P \hat{P}}(P, \hat{P} ; 0)\right]-2(k+2 u)\left(\frac{1}{b+2 f}\right)^{2} \sigma_{\eta}^{2} .
\end{aligned}
$$

Recall that the maximized value of nohat's zero-disturbance profits depends only on which instrument is chosen by hat and not on which instrument is chosen by nohat as stated in equations (3). Equations (23) and equations (3) together imply that

$$
\begin{aligned}
& \max _{Q}\left[E \Pi^{Q \hat{Q}}(Q, \hat{Q} ; \eta)\right]-\max _{P}\left[E \Pi^{P \hat{Q}}(P, \hat{Q} ; \eta)\right]=2 k\left(\frac{1}{b}\right)^{2} \sigma_{\eta}^{2}, \\
& \max _{Q}\left[E \Pi^{Q \hat{P}}(Q, \hat{P} ; \eta)\right]-\max _{P}\left[E \Pi^{P \hat{P}}(P, \hat{P} ; \eta)\right]=2(k+2 u)\left(\frac{1}{b+2 f}\right)^{2} \sigma_{\eta}^{2}
\end{aligned}
$$


Our analysis of instrument choice is based on equations (24). If hat chooses $\hat{Q}$ as an instrument, nohat's expected profit is higher when $Q$ or $P$ is chosen as an instrument depending on whether $k$ is positive or negative, that is, depending on whether nohat's marginal cost is increasing or decreasing in his own output. If hat chooses $\hat{P}$ as an instrument, nohat's expected profit is higher when $Q$ or $P$ is chosen as an instrument depending on whether

$$
k+2 u \gtrless 0 .
$$

\section{The Klemperer and Meyer Result}

Klemperer and Meyer (1986) consider the case of independent costs $(u=0)$ and find that there is always a unique equilibrium. In this case, if hat chooses $\hat{P}$ as an instrument, nohat's expected profit is higher when $Q$ or $P$ is chosen as an instrument depending on whether $k$ is positive or negative according to the condition in equation (25). It follows that no matter whether hat chooses $\hat{Q}$ or $\hat{P}$ as an instrument, nohat's expected profit is higher when $Q$ or $P$ is chosen as an instrument depending on whether $k$ is positive or negative. A similar argument can be used to establish that in the case of independent costs no matter whether nohat chooses $Q$ or $P$ as an instrument, hat's expected profit is higher when $\hat{Q}$ or $\hat{P}$ is chosen as an instrument depending on whether $k$ is positive or negative. Therefore, if $k$ is posilive, there is a unique equilibrium in which $Q$ and $\hat{Q}$ are chosen as the instruments, 
and if $k$ is negative there is unique equilibrium in which $P$ and $\hat{P}$ are chosen as the instruments.

The Klemperer and Meyer result can be explained graphically using Figure $3 .^{16}$

No matter whether hat chooses $\hat{Q}$ or $\hat{P}$ as an instrument, nohat faces a residual demand curve. The slope and location of the residual demand curve are different depending on which instrument hat chooses, and the location of the residual demand curve depends on the value at which hat's instrument is set. However, the slope and location of the residual demand curve do not affect the argument made below, so $D$ and $M R$ can be thought of as representing the zero-disturbance residual demand curve and residual marginal revenue curve, respectively, for a particular value of either of hat's possible instruments. As a benchmark, we consider the case of constant marginal costs $(k=0)$ represented by $M C$ in Figure 3. Nohat's profits in the zero-disturbance equilibrium are represented by the difference between the area under $M R$ and the area under $M C$ between zero and $Q_{1}$.

Suppose nohat's residual demand curve shifts up from $D$ to $D^{\prime}$. If nohat chooses $Q$ as an instrument, profits increase by the amount of the difference between the areasi under $M R$ and $M R^{\prime}$ between the origin and $Q_{1}$. If nohat chooses $P$ as an instrument, the change in profits has three components: (1) an increase equal to the increase that occurs when nohat chooses $Q$ as an instrument; (2) an increase equal to the difference between the areas under $M R^{\prime}$ and $M C$ between $Q_{1}$ and $\tilde{Q}$, the ex post optimal quantity, represented by the lined

\footnotetext{
${ }^{16}$ Figure 3 is constructed in the same way as the figures used by Klemperer and Meyer (1986) to illustrate their result.
} 


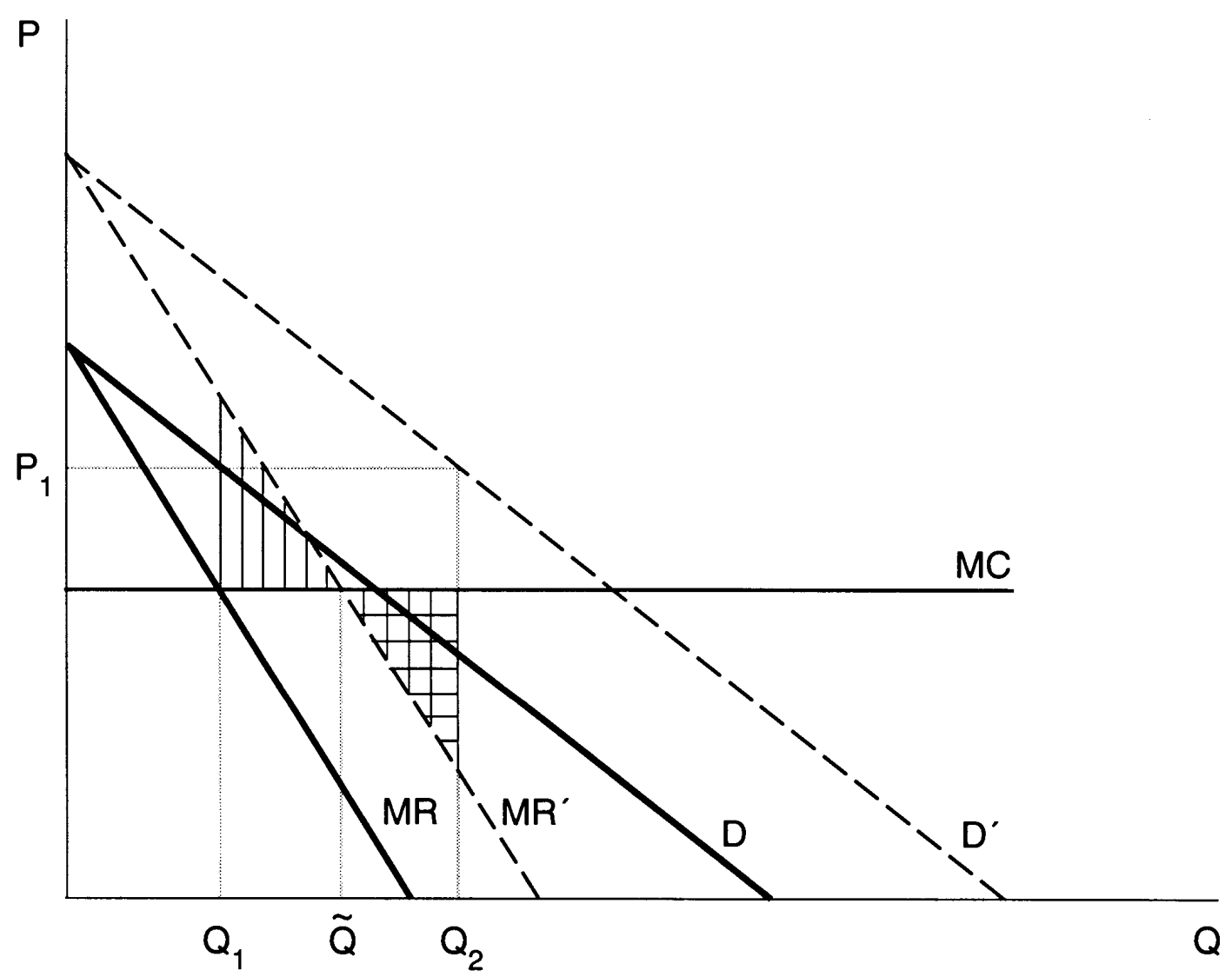

Figure 3 
triangle in Figure 3; and (3) a decrease equal to the difference between the areas tnder $M R^{\prime}$ and $M C$ between $\tilde{Q}$ and $Q_{2}$, the quantity at which $P=P_{1}$, represented by the hatched triangle in Figure 3. Nohat prefers $Q$ as an instrument, is indifferent between $Q$ and $P$, or prefers $P$ as an instrument depending on whether the lined triangle is smaller than, equal to, or larger than the hatched triangle.

The lined triangle is the same size as the hatched triangle if marginal cost is constant, the case represented by $M C$ in Figure 3. The two triangles are similar. ${ }^{17}$ Furthermore, $\tilde{Q}-Q_{1}=Q_{2}-\tilde{Q} \cdot Q_{2}-Q_{1}$ is the increase in $Q$ required to keep $P=P_{1} \cdot \tilde{Q}-Q_{1}$ is the increase in $Q$ required to keep marginal revenue constant. Since the residual demand curve is linear, $\tilde{Q}-Q_{1}$ is half of $Q_{2}-Q_{1}$. It follows that the lined triangle is sinaller or larger than the hatched triangle depending on whether marginal cost is rising $(k>0)$ or falling $(k<0)$. Thus, nohat prefers $Q$ as an instrument if $k>0$ and prefers $P$ a.s an instrument if $k<0$.

There is a striking result not emphasized by Klemperer and Meyer. As shown above, in the case of independent costs $(u=0)$ with no uncertainty, profits are larger in the $Q \hat{Q}$ equilibrium than in the $P \hat{P}$ equilibrium. In the presence of the smallest amount of uncertainty with $k<0$, there is a unique equilibrium in which $P$ and $\hat{P}$ are chosen as the

\footnotetext{
${ }^{17}$ They are formed by two parallel lines cutting two intersecting straight lines on opposite sides of their intersection.
} 
instruments. That is, for some parameter values, with the smallest amount of uncertainty the symmetric instrument pair chosen in the unique equilibrium is the one that yields lower profits with no uncertainty.

\section{Joint Costs}

In the case of joint costs considered in this paper, for some parameter values there is a unique tquilibrium but for others there are two equilibria. The crucial difference between the case of independent costs and the case of joint costs is that in the latter, each duopolist's marginal cost depends on the output of the other.

If hat chooses $\hat{Q}$ as an instrument, the results for nohat's instrument choice are the same with joint costs as with independent costs: nohat prefers $Q$ or $P$ as an instrument depending on whether $k$ is greater than or less than zero. A shift in $D$ leaves nohat's marginal cost curve unaffected even though $\hat{Q}$ enters nohat's marginal costs because $\hat{Q}$ is kept fixed. Therefore, the graphical analysis above for the case of independent costs applies.

Hcwever, if hat chooses $\hat{P}$ as an instrument, the results for nohat's instrument choice are different with joint costs than with independent costs according to the condition in equation (25). Suppose nohat's costs rise with the square of own output $(k>0)$. If each duopolist's costs rise with the product of outputs $(u>0)$, nohat definitely prefers $Q$ as an instrumen: according to the condition in equation (25). However, if each duopolist's costs fall with the product of outputs $(u<0)$, nohat prefers $Q$ or $P$ as an instrument depending on whether $k+2 u \gtrless 0$. Now suppose that $k<0$. If $u<0$, nohat definitely prefers $\boldsymbol{P}$ as 
instrument. However, if $u>0$, nohat prefers $P$ or $Q$ as an instrument depending on whether $k+2 u \lesseqgtr 0$. It can be shown that if nohat chooses $P$ as instrument, the analysis of this paragraph applies mutatis mutandi to hat's instrument choice. It follows that there is a unique equilibrium or two equilibria depending on whether

$$
k(k+2 u) \gtrless 0 .
$$

The logic behind the results of the preceding paragraph can be explained graphically using Figure 4 drawn for the case in which $u<0, k=0$, and hat chooses $\hat{P}$ as an instrument. Suppose nohat's residual demand curve shifts from $D$ to $D^{\prime}$. Recall that the same disturbance affects the demand curve of each duopolist. In order to keep $\hat{P}$ from rising, hat must raise $\hat{Q}$. The increase in $\hat{Q}$ lowers nohat's marginal cost from $M^{\prime} C$ to $M C^{\prime}$. This shift down in the marginal cost schedule in Figure 4 is the only difference between Figures 3 and 4 . Since we know that with $k=0$ and independent costs, nohat is indifferent between $P$ and $Q$ as instruments because the lined triangle is the same size as the hatched triangle in Figures 3, we can focus on the additional changes in nohat's profits that: arise in the case of joint costs. If nohat chooses $Q$ as an instrument, the additional increase in nohat's profits is equal to the difference between the areas under $M C$ and $M C^{\prime}$ between the origin and $Q_{1}$. If nohat chooses $P$ as an instrument, the additional increase in profits has two components: (1) the additional increase that would occur if nohat chose $Q$ as an instrument and (2) the additional increase corresponding to the difference between the areas 


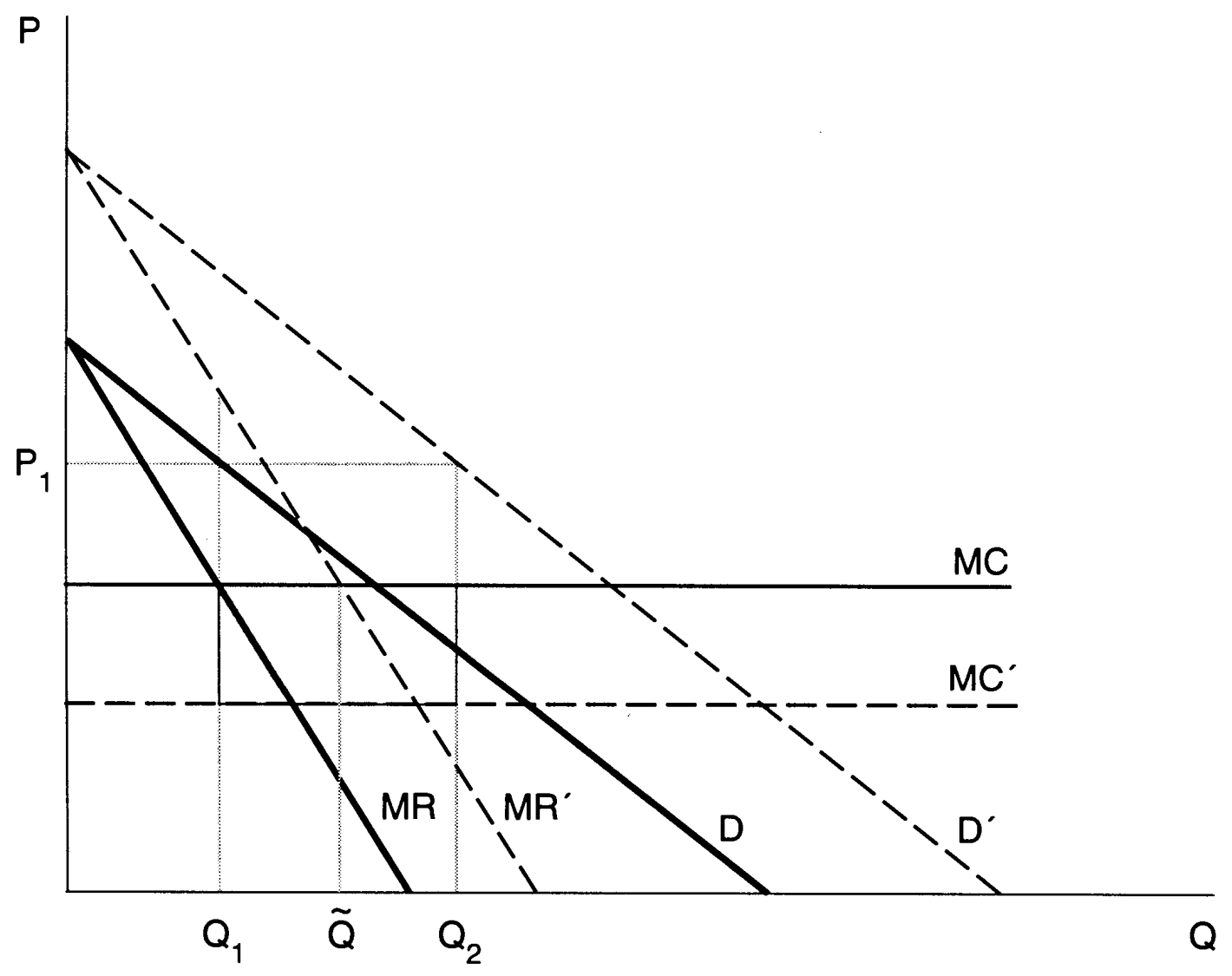

Figure 4 
under $M C$ and $M C^{\prime}$ between $Q_{1}$ and $Q_{2}$ represented by the shaded area in Figure 4 . It follows that nohat's profits are higher if $P$ is chosen as an instrument.

Since with $u<0$ and $k=0$ nohat prefers $P$ as an instrument, it makes sense that nohat will also prefer $P$ as an instrument if $u<0$ and $k$ is positive but small enolıgh that $k+2 u<0$ as stated in equation (25). Since nohat prefers $Q$ as an instrument with $u>0$ and $k=0$, it makes sense that nohat will also prefer $Q$ as an instrument if $u>0$ and $k$ is negative but small enough in absolute value that $k+2 u>0$ as stated in equation (25).

What conclusions can be drawn regarding the number of equilibria in a differentiated duopoly game with joint costs? If $k(k+2 u)>0$, there is a unique equilibrium in which either $Q$ and $\hat{Q}$ (if $k>0, k+2 u>0$ ) or $P$ and $\hat{P}$ (if $k<0, k+2 u<0$ ) are chosen as instruments. However, if $k(k+2 u)<0$, there are two equilibria, one in which $Q$ and $\hat{Q}$ are chosen as instruments and one in which $P$ and $\hat{P}$ are chosen as instruments. That is, for some parameter values there is only one equilibrium, but for others there are two.

It should come as no surprise that with joint costs just as with independent costs, for some parameter values there is a unique equilibrium in which the symmetric instrument pair chosen is the one that yields lower profits with no uncertainty. For example, as shown above, with no uncertainty, profits are always higher in the $Q \hat{Q}$ equilibrium than in the $P \hat{P}$ equilibrium. In the presence of the smallest amount of uncertainty with $k<0$ and $k+2 u<0$, there is a unique equilibrium in which $P$ and $\hat{P}$ are chosen as the instruments. 


\section{A Macroeconomic Example: Inflation Bias and Uncertain Money Demands}

In the macroeconomic example, we use a two-country version of the Kydland-Prescott (1977) and Barro-Gordon (1983) inflation-bias model similar to the one in Henderson and Zhu (199()). First, we lay out a model with $(\log )$ linear relations summarizing private sector behavior, quadratic inflation-bias preferences for countries, and an additive velocity disturbance that affects both money demands. Then, we discuss the multiplicity of equilibria with no uncertainty. Finally, we consider the implications of introducing uncertainty in the form of the additive velocity disturbance.

\section{A. The Mlodel}

\section{Private Sector Behavior}

We use a model with two countries, nohat and hat. ${ }^{18}$ Each country specializes in the production of a different good, and the natural rates of output in the two countries are the same when they are measured in the same good. All the variables of the model are logarithms except for the interest rates. Variables with hats over them are hat country variables. Time subscripts are suppressed wherever possible.

According to the production functions, outputs $(y, \hat{y})$ are increasing functions of employments $(n, \hat{n})$ :

${ }^{18}$ Our model is identical to the one used in Chapter 2 of Canzoneri and Henderson (1991). Leterministic and stochastic special cases of this model are used in Canzoneri and Henderson (1989) and Henderson and Zhu (1990), respectively. A similar model with no capital mobility is used in Canzoneri and Henderson (1988). 


$$
y=\bar{y}+(1-\alpha) n, \quad \hat{y}=\bar{y}+(1-\alpha) \hat{n},
$$

where $0<\alpha<1$ and $\bar{y}=-\ln (1-\alpha)$. Units are defined so that the natural rate of employment is equal to zero in each country, so $\bar{y}$ is the natural rate of output in each country.

In order to maximize profits, firms employ labor up to the point at which marginal products of labor are equal to real product wages:

$$
w-p=-\alpha n, \quad \hat{w}-\hat{p}=-\alpha \hat{n} .
$$

Marginal products decrease as employments increase. Real product wages are nominal wages $(w, \hat{w})$ minus product prices $(p, \hat{p})$. Equations (28) can be solved for output prices:

$$
p=w+\alpha n, \quad \hat{p}=\hat{w}+\alpha \hat{n}
$$

Before markets meet each period, workers and firms enter into wage contracts that specify nominal wages and employment rules. We explain how nominal wages are determined later when we discuss the game between the countries; at this point we take them as given. Workers agree to supply whatever quantity of labor firms want at the nominal wages specified in the contracts.

Consumer price levels $(q, \hat{q})$ are given by

$$
\begin{aligned}
& q=(1-\beta) p+\beta(e+\hat{p})=p+\beta z, \\
& \hat{q}=\beta(p-e)+(1-\beta) \hat{p}=\hat{p}-\beta z,
\end{aligned}
$$


where $0<\beta<1$ is the average propensity to import in each country, the exchange rate $(e)$ is the nohat currency price of hat currency, and the relative price of the foreign good or real exchange rate $(z)$ is

$$
z=e+\hat{p}-p
$$

Eich country's money is held only by the residents of that country. Money markets are in equilibrium when money supplies $(m, \hat{m})$ satisfy simple quantity-theory equations:

$$
m+v+\bar{y}=p+y, \quad \hat{m}+v+\bar{y}=\hat{p}+\hat{y} .
$$

For algebraic simplicity, the expected value of the logarithm of velocity has been set equal to $\bar{y}$ in each country. The world velocity disturbance $(v)$ is identically and independently distributed with a zero mean.

Residents of each country may hold bonds denominated in both currencies. They regard bonds denominated in the two currencies as perfect substitutes, so they will hold positive amounts of both kinds of bonds only when the nominal interest rate on nohatcurrency bonds $(i)$ is equal to the nominal interest rate on hat-currency bonds $(\hat{i})$ plus the expected rate of depreciation:

$$
i=\hat{i}+e_{+1}-e
$$

where a variable with a +1 subscript is the value of that variable expected to prevail next period based on today's information. In what follows we refer to nominal interest rates simply as interest rates. The expected real interest rates for residents of the two countries are 


$$
r=i-q_{+1}+q, \quad \hat{r}=\hat{i}-\hat{q}_{+1}+\hat{q} .
$$

Log-linearizing the demands for the two goods in the neighborhood of the rlatural rates of output yields

$$
\begin{aligned}
& y=\bar{y}+\delta z+(1-\beta) \varepsilon(y-\bar{y})+\beta \varepsilon(\hat{y}-\bar{y})-(1-\beta) \gamma r-\beta \gamma \hat{r}, \\
& \hat{y}=\bar{y}-\delta z+\beta \varepsilon(y-\bar{y})+(1-\beta) \varepsilon(\hat{y}-\bar{y})-\beta \gamma r-(1-\beta) \gamma \hat{r} .
\end{aligned}
$$

Demands for both goods increase with both outputs. Residents of each country increase spending by the same fraction $(0<\varepsilon<1)$ of increases in output. The marginal propensity to import is equal to the average propensity to import of $\beta$ in each country. Demands for both goods decrease with expected real interest rates $(r, \hat{r})$. Residents of each country decrease spending by the same amount $(\gamma)$ for each percentage point increase in the expected real interest rate available to them. Depreciation of the real exchange rate shifts world demand from foreign goods to home goods. ${ }^{19}$

We assume that countries care about employments $(n, \hat{n})$ and inflations $(\pi, \hat{\pi})$, where

$$
\pi=q-q_{-1}, \quad \hat{\pi}=\hat{q}-\hat{q}_{-1},
$$

so we solve the model for these variables. Since the countries are symmetric, we need

${ }^{19}$ It is assumed (1) that trade is balanced in zero-disturbance equilibrium and (2) that the response of home spending measured in home goods to changes in the real exchange rate is the same as the response of foreign spending measured in foreign goods. These two assumptions are sufficient to insure that the responses of demands for the two goods to changes in the real exchange rate are equal and opposite in sign. 
explain in detail the solution for only one country, say nohat. The solutions are different when different variables are chosen as policy instruments. Each country can choose either the money supply or the interest rate as an instrument, so there are four regimes: the $m \hat{m}, i \hat{i}, n \hat{i}$, and $i \hat{m}$ regimes. We also calculate the inflation-employment tradeoffs faced by the count:ies. The tradeoff faced by a country depends not on which variable it chooses as its instrurnent but on which variable the other country chooses as its instrument.

In the $m \hat{m}$ regime, the reduced forms for employments can be obtained by substituting equations (27) and (29) into equations (32):

$$
n=m+v-w, \quad \hat{n}=\hat{m}+v-\hat{w} \text {. }
$$

Nohat employment rises one for one with the nohat money supply and is unaffected by the hat money supply. These results depend crucially on two key features of our quantity-theory formulation: the nohat interest rate does not enter nohat money demand, and the real income elasticity of nohat money demand is equal to one. These features imply that increases in the nohat money supply must be matched by increases in nohat nominal income. Nohat nominal income increases one for one with increases in nohat employment and is unaffected by increases in the hat money supply.

Obtaining solutions for inflation rates takes somewhat longer. According to equations (30), each country's consumer price level can be written as a weighted sum of that country's product price and the real exchange rate. Product prices are obtained by substitutirg equations (37) into equations (29):

Increases in $m$ raise $n$ thereby lowering the nohat marginal product of labor, so $p$ must rise 


$$
p=\alpha(m+v-w)+w, \quad \hat{p}=\alpha(\hat{m}+v-\hat{w})+\hat{w}
$$

to reduce the nohat real product wage.

The real exchange rate is obtained in several steps. Subtracting the second equation in equations (35) from the first and solving for $z$ yields

$$
2 \delta z=[1-(1-2 \beta) \varepsilon][(y-\bar{y})-(\hat{y}-\bar{y})]+(1-2 \beta) \gamma(r-\hat{r}) .
$$

Subtracting the second equation in equations (34) from the first and making use of equations (30), (31), and (33) yields

$$
r-\hat{r}=(1-2 \beta)\left(z_{+1}-z\right)=-(1-2 \beta) z
$$

where $z_{+1}=0$ because we assume that there are no speculative bubbles. ${ }^{20}$ Substituting equation (40) into equation (39) and solving for $z$ yields

$$
\begin{aligned}
& z=\left(\frac{\rho}{1-\alpha}\right)[(y-\bar{y})-(\hat{y}-\bar{y})], \\
& \rho=\frac{(1-\alpha)[1-(1-2 \beta) \varepsilon]}{2 \delta+(1-2 \beta)^{2} \gamma} .
\end{aligned}
$$

Substituting equations (37) into equations (27) and substituting the resulting equations into equation (41) yields

Increases in a country's money supply raise its employment and, therefore, its output.

\footnotetext{
${ }^{20}$ For proof that the assumption that there are no speculative bubbles implies that $z_{+1}=0$ see Appendix A of Canzoneri and Henderson (1991).
} 


$$
z=\rho[(m+v-w)-(\hat{m}+v-\hat{w})] .
$$

Increases in nohat output and decreases in hat output increase the difference between the excess supply of the nohat good and the excess supply of the hat good, so the nohat currency must dep:eciate in real terms.

The reduced forms for price levels and inflation rates are obtained by substituting equations (38) and (42) into equations (30):

$$
\begin{aligned}
& \pi=q=w+(\alpha+\beta \rho)(m+v-w)-\beta \rho(\hat{m}+v-\hat{w}), \\
& \hat{\pi}=\hat{q}=\hat{w}-\beta \rho(m+v-w)+(\alpha+\beta \rho)(\hat{m}+v-\hat{w}),
\end{aligned}
$$

where we have set last period's price levels equal to zero $\left(q_{-1}=\hat{q}_{-1}=0\right)$ so that price levels and inflation rates are the same thing in the current period. Increases in the nohat money supply ra se the price of nohat output and cause real depreciation of the nohat currency, so they increase nohat inflation and decrease hat inflation.

Fcr the $m \hat{m}$ regime we collect together the reduced forms for employments and inflations: and present the inflation-employment tradeoffs:

$$
\begin{aligned}
& n^{m \hat{m}}=m+v-w, \\
& \hat{n}^{m \hat{m}}=\hat{m}+v-\hat{w},
\end{aligned}
$$




$$
\begin{aligned}
& \pi^{m \hat{m}}=w+(\alpha+\beta \rho)(m+v-w)-\beta \rho(\hat{m}+v-\hat{w}), \\
& \hat{\pi}^{m \hat{m}}=\hat{w}-\beta \rho(m+v-w)+(\alpha+\beta \rho)(\hat{m}+v-\hat{w}), \\
& \tau^{m \hat{m}}=\frac{\pi_{m}^{m \hat{m}}}{n_{m}^{m \hat{m}}}=\alpha+\beta \rho, \quad \hat{\tau}^{m \hat{m}}=\frac{\hat{\pi}_{\dot{m}}^{m \hat{m}}}{\hat{n}_{\hat{m}}^{m \hat{m}}}=\alpha+\beta \rho,
\end{aligned}
$$

where $n^{\hat{j}}, \hat{n}^{\hat{j}}, \pi^{\hat{j}}$, and $\hat{\pi}^{j \hat{j}}$ represent the solutions for $n, \hat{n}, \pi$, and $\hat{\pi}$ under the $j \hat{j}$ regime, respectively, and where $\tau^{i \hat{j}}$ represents the inflation-employment tradeoff for nohat 'when hat chooses $\hat{j}$ as its instrument and $\hat{\tau} \hat{j}$ represents the inflation-employment tradeoff for: hat when nohat chooses $j$ as its instrument.

It is useful to derive solutions for (expected) real interest rates in the $m \hat{m}$ regime. Adding the two equations in equations (35) and rearranging yields

$$
\begin{aligned}
r+\hat{r} & =-\left(\frac{2 \phi}{1-\alpha}\right)[(y-\bar{y})+(\hat{y}-\bar{y})] \\
\phi & =\left(\frac{1-\varepsilon}{2 \gamma}\right)(1-\alpha) .
\end{aligned}
$$

Eliminating $z$ from equation (40) using equation (41) yields 


$$
\begin{aligned}
r-\hat{r} & =-\left(\frac{2 \theta}{1-\alpha}\right)[(y-\bar{y})-(\hat{y}-\bar{y})], \\
\theta & =\left(\frac{1-2 \beta}{2}\right) \rho .
\end{aligned}
$$

Using equations (47) and (48) to solve for $r$ and $\hat{r}$ in terms of $y-\bar{y}$ and $\hat{y}-\bar{y}$, and using equations (27) and (37) to eliminate $y-\bar{y}$ and $\hat{y}-\bar{y}$ yields

$$
\begin{aligned}
& r=i-\pi_{+1}=-(\theta+\phi)(m+v-w)+(\theta-\phi)(\hat{m}+v-\hat{w}), \\
& \hat{r}=\hat{i}-\hat{\pi}_{+1}=(\theta-\phi)(m+v-w)-(\theta+\phi)(\hat{m}+v-\hat{w}) .
\end{aligned}
$$

Inzreases in the sum of and the difference between nohat's output and hat's output increase the sum of and the difference between excess supplies of the two goods, respectively. Therefore, it is logical that $r+\hat{r}$ is negatively related to the sum of outputs and that $r-\hat{r}$ is negatively related to the difference between outputs as shown in equations (47) and (48), respectively.

$r$ can be expressed as half the sum of $r+\hat{r}$ and $r-\hat{r}$. Increases in $m$ lower $r$ because they increase both the sum of outputs and the difference between outputs. $\hat{r}$ can be expressed as half the difference between $r+\hat{r}$ and $r-\hat{r}$. Under our assumption that $\theta>\phi$, an increase in $m$ raises $\hat{r}$ because the decrease in $r-\hat{r}$ caused by the increase in the difference between outputs is greater in absolute value than the decrease in $r+\hat{r}$ caused by the inc rease in the sum of outputs. 
A decrease in $i$ with no change in $\hat{i}$ is achieved by increasing $m$ and increasing $\hat{m}$ by less. $\quad \hat{m}$ must increase because an increase in $m$ with $\hat{m}$ held constant causes $r$ to fall and $\hat{r}$ to rise. However, the increase in $\hat{m}$ must be less than the increase in $m$ bezause equal increases in $m$ and $\hat{m}$ lower both $r$ and $\hat{r}$. We assume that $\pi_{+1}$ and $\hat{\pi}_{+1}$ are unaffected by changes in $m$ and $\hat{m}$, so changes in $r$ and $\hat{r}$ are reflected completely in changes in $i$ and $\hat{i}$, respectively.

For what follows it is helpful to use equations (49) to obtain expressions for $m+v-w$ and $\hat{m}+v-\hat{w}$ in terms of $i-\pi_{+1}$ and $\hat{i}-\hat{\pi}_{+1}$ :

$$
\begin{aligned}
& m+v-w=-\left(\frac{\theta+\phi}{4 \theta \phi}\right)\left(i-\pi_{+1}\right)-\left(\frac{\theta-\phi}{4 \theta \phi}\right)\left(\hat{i}-\hat{\pi}_{+1}\right) \\
& \hat{m}+v-\hat{w}=-\left(\frac{\theta-\phi}{4 \theta \phi}\right)\left(i-\pi_{+1}\right)-\left(\frac{\theta+\phi}{4 \theta \phi}\right)\left(\hat{i}-\hat{\pi}_{+1}\right) .
\end{aligned}
$$

In an $i \hat{i}$ regime, the reduced forms for employments and inflation rates and the inflation-employment tradeoffs are

$$
\begin{aligned}
& n^{i \hat{i}}=-\left(\frac{\theta+\phi}{4 \theta \phi}\right)\left(i-\pi_{+1}\right)-\left(\frac{\theta-\phi}{4 \theta \phi}\right)\left(\hat{i}-\hat{\pi}_{+1}\right), \\
& \hat{n}^{\hat{i}}=-\left(\frac{\theta-\phi}{4 \theta \phi}\right)\left(i-\pi_{+1}\right)-\left(\frac{\theta+\phi}{4 \theta \phi}\right)\left(\hat{i}-\hat{\pi}_{+1}\right),
\end{aligned}
$$




$$
\begin{aligned}
& \pi^{i \hat{i}}=-\left(\frac{\theta+\phi}{4 \theta \phi}\right)\left[\alpha+\beta \rho\left(\frac{2 \phi}{\theta+\phi}\right)\right]\left(i-\pi_{+1}\right) \\
& -\left(\frac{\theta+\phi}{4 \theta \phi}\right)\left[\alpha-(\alpha+\beta \rho)\left(\frac{2 \phi}{\theta+\phi}\right)\right]\left(\hat{i}-\hat{\pi}_{+1}\right)+w, \\
& \hat{\pi}^{i \hat{i}}=-\left(\frac{\theta+\phi}{4 \theta \phi}\right)\left[\alpha-(\alpha+\beta \rho)\left(\frac{2 \phi}{\theta+\phi}\right)\right]\left(i-\pi_{+1}\right) \\
& -\left(\frac{\theta+\phi}{4 \theta \phi}\right)\left[\alpha+\beta \rho\left(\frac{2 \phi}{\theta+\phi}\right)\right]\left(\hat{i}-\hat{\pi}_{+1}\right)+\hat{w} . \\
& \tau^{i \hat{i}}=\frac{\pi_{i}^{i \hat{i}}}{n_{i}^{i \hat{i}}}=\alpha+\beta \rho\left(\frac{2 \phi}{\theta+\phi}\right), \quad \hat{\tau}^{\hat{i} \hat{i}}=\frac{\pi_{\hat{i}}^{i \hat{i}}}{n_{\hat{i}}^{i \hat{i}}}=\alpha+\beta \rho\left(\frac{2 \phi}{\theta+\phi}\right) .
\end{aligned}
$$

The solutions for employments and inflation rates are obtained by using equations (50) to eliminate $m+v-w$ and $\hat{m}+v-\hat{w}$ in equations (44) and (45).

The important thing to note here is that nohat's inflation-employment tradeoff is flatter when hat is fixing $\hat{i}$ than when hat is fixing $\hat{m}$; that is $\tau^{i \hat{i}}<\tau^{m \hat{m}}$ if $\theta>\phi$. As explained above, a reduction in $i$ with $\hat{i}$ held constant is achieved by increasing $m$ and increasing $\hat{m}$ by less. Therefore, when hat is fixing $\hat{i}$, the increase in $\pi$ accompanying an increase in $n$ is less be:cause the increase in $\hat{m}$ partially offsets the real depreciation of nohat's currency caused by the increase in $m$.

II an $m \hat{i}$ regime, the solutions for employments and inflation rates and the inflationemployment tradeoffs are 


$$
\begin{gathered}
n^{m \hat{i}}=m+v-w, \\
\hat{n}^{m \hat{i}}=\left(\frac{\theta-\phi}{\theta+\phi}\right)(m+v-w)-\left(\frac{1}{\theta+\phi}\right)\left(\hat{i}-\hat{\pi}_{+1}\right), \\
\pi^{m \hat{i}}=\left[\alpha+\beta \rho\left(\frac{2 \phi}{\theta+\phi}\right)\right](m+v-w)+\left(\frac{\beta \rho}{\theta+\phi}\right)\left(\hat{i}-\hat{\pi}_{+1}\right)+w, \\
\hat{\pi}^{m \hat{i}}=\left[\alpha-(\alpha+\beta \rho)\left(\frac{2 \phi}{\theta+\phi}\right)\right]\left(m+v-w^{2}-\left(\frac{\alpha+\beta \rho}{\theta+\phi}\right)\left(\hat{i}-\hat{\pi}_{+1}\right)+\hat{v},\right. \\
\tau^{m \hat{i}}=\frac{\pi_{m}^{m \hat{i}}}{n_{m}^{m \hat{i}}}=\alpha+\beta \rho\left(\frac{2 \phi}{\theta+\phi}\right), \quad \hat{\tau}^{m \hat{i}}=\frac{\hat{\pi}_{\hat{i}}^{m \hat{i}}}{\hat{n}_{\hat{i}}^{m \hat{i}}}=\alpha+\beta \rho .
\end{gathered}
$$

The solutions for employments and inflation rates are obtained by solving the secorid equation in equations (49) for $\hat{m}+v-\hat{w}$ and using the result to eliminate this variable in e:quations (44) and (45).

In an im regime, the reduced forms for employments and inflations are completely symmetric to the reduced forms in a $m \hat{i}$ regime. Nohat and hat variables are simply interchanged.

The important thing to note here is that a country's choice of instrument does not affect its own inflation-employment tradeoff, but it does affect its opponent's. No matter which instrument nohat chooses, it faces the steep tradeoff, $\tau^{m \hat{m}}=\tau^{i \hat{m}}=\alpha+\beta \rho$, when hat 
fixes $\hat{m}$ and the flat tradeoff, $\tau^{i \hat{i}}=\tau^{m \hat{i}}=\alpha+\beta \rho\left(\frac{2 \phi}{\theta+\phi}\right)$, when hat fixes $\hat{i}$.

\section{Countries' Utility Functions}

The countries' period utility functions are ${ }^{21}$

$$
U=\Psi n-.5 \pi^{2}, \quad \hat{U}=\Psi \hat{n}-.5 \hat{\pi}^{2} .
$$

Each country's period utility is increasing in its employment and decreasing in squared deviations of its inflation from zero. Wage setters want zero employments, and they set the nominal vages, $w$ and $\hat{w}$, to achieve their goals. Why do countries want higher employments than their wage setters? At least two answers have been given: one is that income taxes may distort the labor-leisure decision, making the "natural rate" of employment too low, and another is that monopolistic unions may seek real wages that are too high for the employment of the entire labor force. Expressions for period utilities in terms of instruments, wages, expected inflations, and the disturbance are given in equations (T2.1) through (T2.4) in Table 2.

\section{B. The Mlodel with No Uncertainty}

\section{The Multiplicity of Equilibria}

W:th no uncertainty $\left(\sigma_{v}^{2}=0\right)$ there is a multiplicity of equilibria in the macroeconomic example, just as in the microeconomic example. The method of proof used

\footnotetext{
${ }^{21}$ In the example in Henderson and Zhu (1990) we assume that the countries' period utility funztions are

$$
U=-\Psi(n-\breve{n})^{2}-.5 \pi^{2}, \quad \hat{U}=-\Psi(\hat{n}-\breve{n})^{2}-.5 \hat{\pi}^{2},
$$
}
where $\breve{n}$ is a bliss level of employment above the natural rate of zero. 
in the macroeconomic example is analogous to the method used in the microeconomic example. However, there is an important difference. In the microeconomic example, each duopolist must take into account only the behavior of the other duopolist. All that either of the duopolists needs to know about other agents is summarized in the demands and costs. In contrast, in the macroeconomic example each country must take into account not only the behavior of the other country but also the behavior of the wage setters and the valves of expected inflation in both countries. As in the microeconomic example, since the players are symmetric we need consider in detail the behavior of only one of them, say nohat.

If hat chooses $\hat{j}$ as an instrument and sets it at a particular value, wage setters set $w$ and $\hat{w}$ at particular values, and $\pi_{+1}$ and $\hat{\pi}_{+1}$ are given, the maximum value of nohat's zerodisturbance utility is the same no matter which variable it chooses as an instrument:

$$
\max _{m}\left[U^{m \hat{j}}\left(m, \hat{j}, w, \hat{w}, \pi_{+1}, \hat{\pi}_{+1} ; 0\right)\right]=\max _{i}\left[\Pi^{i \hat{j}}\left(i, \hat{j}, w, \hat{w}, \pi_{+1}, \hat{\pi}_{+1} ; 0\right)\right]
$$

For given values for $\hat{j}, w, \hat{w}, \pi_{+1}$, and $\hat{\pi}_{+1}$, there is a one to one relationship between $m$ and $i$.

It follows that there are four equilibria in which the instrument pairs are $m$ and $\hat{m}, i$ and $\hat{m}, m$ and $\hat{i}$, and $i$ and $\hat{i}$ respectively. We refer to these equilibria as the $m i \hat{n}, i \hat{m}$, $m \hat{i}$, and $i \hat{i}$ equilibria, respectively. As an example, we establish that there is an im 
equilibrium conditional on values for $w, \hat{w}, \pi_{+1}$, and $\hat{\pi}_{+1}$. The determination of the equilibritum values of $w, \hat{w}, \pi_{+1}$, and $\hat{\pi}_{+1}$ is explained below. Begin with the expressions for nohat's and hat's utility when $i$ and $\hat{m}$ are chosen as instruments, where the expression for nohat is equation (T2.2) in Table 2 and the expression for hat is analogous to equation (T2.3) in Table 2. Set both the first derivative of nohat's utility function with respect to $i$ and the first derivative of hat's utility function with respect to $\hat{m}$ equal to zero. This pair of first order conditions is a pair of simultaneous linear equations in $i$ and $\hat{m}$. It remains to demonstrate that the solution to this pair of first order conditions is an equilibrium. Given the solution for $\hat{m}$, the solution for $i$ is the best response for nohat given that it chooses $i$ as an instrument. Likewise, given the solution for $i$, the solution for $\hat{m}$ is the best response for hat given that it chooses $\hat{m}$ as an instrument. As we argue in the preceding paragraph, nohat can do no better by choosing $m$ as an instrument. Likewise, hat can do no better by choosing $\hat{i}$ as an instrument. Therefore, the solution to the pair of first order conditions when $i$ and $\hat{m}$ are chosen as instruments is an equilibrium. In a similar manner, it can be established that there are $m \hat{m}, m \hat{i}$ and $i \hat{i}$ equilibria conditional on values for $w, \hat{w}, \pi_{+1}$, and $\hat{\pi}_{+1}$.

\section{The Comparison of Utilities at Five Points}

In this subsection, we compare utilities at the symmetric efficient point with those in the four noncooperative equilibria, the $m \hat{m}, i \hat{m}, m \hat{i}$, and $i \hat{i}$ equilibria. 
The Efficient Point. As in the microeconomic example, we use as a standard of comparison the symmetric efficient point, the point at which the simple sum of the utilities of the countries is maximized subject to the constraint that employments are zero, anc refer to it as "the" efficient point.

Setting up the maximization problem and deriving the first order conditions are straightforward exercises, but little insight is gained by performing them. It is obvious from inspection of the countries' utility functions that if employments are constrained to be equal to zero, then in order to maximize the sum of their utilities the countries should choose their policy instruments so that their inflation rates are equal to zero.

The Four Noncooperative Equilibria. Now we derive expressions for countries' utilities in the four noncooperative equilibria, the $m \hat{m}, i \hat{m}, m \hat{i}$, and $i \hat{i}$ equilibria. As part of the derivation, we explain how $w, \hat{w}, \pi_{+1}$, and $\hat{\pi}_{+1}$ are determined.

The first order conditions for the countries are

$$
U_{j}^{i \hat{j}}=\Psi n_{j}^{i \hat{j}}-\pi \pi_{j}^{i \hat{j}}=0, \quad \hat{U}_{\hat{j}}^{\hat{j}}=\Psi \hat{n}_{\hat{j}}^{\hat{j}}-\hat{\pi} \hat{\pi}_{\hat{j}}^{\hat{j}}=0,
$$

and the reduced forms for $n, \hat{n}, \pi$, and $\hat{\pi}$ for each instrument pair are given above.

Equilibrium inflation rates are obtained by solving equations (59) for $\pi$ and $\hat{\pi}$ :

$$
\pi^{i \hat{j}}=\Psi\left[\frac{\pi_{j}^{j \hat{j}}}{n_{j}^{i \hat{j}}}\right]^{-1}=\frac{\Psi}{\tau}, \quad \hat{\pi}^{j \hat{j}}=\Psi\left[\frac{\hat{\pi}_{\hat{j}}^{i \hat{j}}}{\hat{n}_{\hat{j}}^{\hat{j} \hat{j}}}\right]^{-1}=\frac{\Psi}{\hat{\tau}^{i \hat{j}}} .
$$

If the instruments chosen imply flat inflation-employment tradeoffs, inflations must be high; if 
the instr.ments chosen imply steep tradeoffs, inflations can be low.

Wage setters know the equilibrium values of $\pi$ and $\hat{\pi}$ and the reduced forms for $n$, $\hat{n}, \pi$, and $\hat{\pi}$, so they can set money wages, $w$ and $\hat{w}$, just high enough that the countries will give them the zero employments they want. Let $j$ and $\hat{j}$ be the instruments the countrie:s have chosen. For given values of $\pi_{+1}$ and $\hat{\pi}_{+1}$, the wage setters can calculate the values of $w, \hat{w}, j$ and $\hat{j}$ that are consistent with the conditions that $\pi$ and $\hat{\pi}$ be equal to the equilibrium values given in equations (60) and with the two additional conditions that $n$ and $\hat{n}$ be equal to zero. Wage setters then set $w$ and $\hat{w}$ equal to the values they have calculated. Given these values of $w$ and $\hat{w}$, the countries must set $j$ and $\hat{j}$ equal to the values calculated by wage setters in order to achieve the equilibrium values of $\pi$ and $\hat{\pi}$. Cinly $i$ and $\hat{i}$ depend on the given values of $\pi_{+1}$ and $\hat{\pi}_{+1}$. We assume that the private sector expects that the countries' preferences will be the same in all future periods and, the efore, sets $\pi_{+1}$ and $\hat{\pi}_{+1}$ equal to the values of $\pi$ and $\hat{\pi}$ given by equations (60) in each period.

T'he equilibrium utilities for the countries are

$$
U^{j \hat{j}}(j, \hat{j} ; 0)=-.5\left[\frac{\Psi}{\tau^{i \hat{j}}}\right]^{2}, \quad \hat{U}^{\hat{j}}(j, \hat{j} ; 0)=-.5\left[\frac{\Psi}{\hat{\tau} \hat{j}}\right]^{2},
$$

where $U^{i \hat{j}}(j, \hat{j} ; 0)$ and $\hat{U}^{\hat{j}}(j, \hat{j} ; 0)$ represent the zero-disturbance utilities of nohat and hat 
when nohat chooses $j$ and hat chooses $\hat{j}$. Each country's utility depends on its inflationemployment tradeoff, a tradeoff imposed on it by the other country's choice of instrument, and on the employment weight in its own utility function but not on the employmert weight in the other country's utility function. ${ }^{22}$ For a given value of its employment weight, the steeper a country's inflation-employment tradeoff, the lower its inflation rate and the higher its utility.

\section{The Implications of Introducing an Additive Disturbance to Money Demands}

In this section we consider the implications of introducing uncertainty into our monetary policy game in the form of an additive velocity disturbance that affects both money demands $\left(\sigma_{v}^{2}>0\right)$. For some parameter values there is a unique equilibrium but for others there are two equilibria.

Recall that we assume that countries' preferences are quadratic, that the behavior of the private sector can be summarized by $(\log )$ linear equations, and that the only kind of uncertainty is an additive velocity disturbance that affects both money demands. These assumptions have the familiar implication that nohat's expected utility for values of an instrument pair $j$ and $\hat{j}$ can be written as the sum of nohat's zero-disturbance utility for those values of that instrument pair and a linear function of the variance of the disturbance:

\footnotetext{
${ }^{22}$ Suppose, for example, that the weight on employment in hat's utility function is $\hat{\Psi}>\Psi$. $\hat{\pi}$ will be greater than $\pi$, but nohat will be unaffected, as long as hat ccntinues to choose the same instrument. The differential between inflation rates is matched exactly by an exchange rate change.
} 


$$
E\left[U^{\hat{j}}\left(j, \hat{j}, w, \hat{w}, \pi_{+1}, \hat{\pi}_{+1} ; v\right)\right]=U^{\hat{j}}\left(j, \hat{j}, w, \hat{w}, \pi_{+1}, \hat{\pi}_{+1} ; 0\right)+\ell^{\hat{j}}\left(\sigma_{v}^{2}\right)
$$

where the linear function $\ell^{\hat{j}}\left(\sigma_{v}^{2}\right)$ depends only on which instrument pair is chosen and not on the values at which the instruments are set.

Consequently, the maximized value of nohat's expected utility for instrument $j$ given a value of instrument $\hat{j}$ can be written as the sum of the maximized value of nohat's zerodisturbance utility for instrument $j$ given that value of instrument $\hat{j}$ and the same linear function of the variance of the disturbance:

$$
\max _{j} E\left[U^{\dot{j}}\left(j, \hat{j}, w, \hat{w}, \pi_{+1}, \hat{\pi}_{+1} ; v\right)\right]=\max _{j}\left[U^{\hat{j}}\left(j, \hat{j}, w, \hat{w}, \pi_{+1}, \hat{\pi}_{+1} ; 0\right)\right]+\ell^{\hat{j}}\left(\sigma_{v}^{2}\right)
$$

Evaluating $\ell^{m \hat{m}}\left(\sigma_{v}^{2}\right), \ell^{i \hat{m}}\left(\sigma_{v}^{2}\right), \ell^{m \hat{i}}\left(\sigma_{v}^{2}\right)$, and $\ell^{i \hat{i}}\left(\sigma_{v}^{2}\right)$ using equations (T2.1), (T2.2),

(T2.3), and (T2.4) and inserting them into the appropriate version of equation (63) yields

$$
\begin{aligned}
\max _{m}\left[E U^{m \hat{m}}(m, \hat{m} ; v)\right] & =\max _{m}\left[U^{m \hat{m}}(m, \hat{m} ; 0)\right]-.5 \alpha^{2} \sigma_{v}^{2}, \\
\max _{i}\left[E U^{i \hat{m}}(i, \hat{m} ; v)\right] & =\max _{i}\left[U^{i \hat{m}}(i, \hat{m} ; 0)\right]-.5\left[\alpha-(\alpha+\beta \rho)\left(\frac{2 \phi}{\theta+\phi}\right)\right]^{2} \sigma_{v}^{2}, \\
\max _{m}\left[E U^{m \hat{i}}(m, \hat{i} ; v)\right] & =\max _{m}\left[U^{m \hat{i}}(m, \hat{i} ; 0)\right]-.5\left[\alpha+\beta \rho\left(\frac{2 \phi}{\theta+\phi}\right)\right]^{2} \sigma_{v}^{2}, \\
\max _{i}\left[E U^{i \hat{i}}(i, \hat{i} ; v)\right] & =\max _{i}\left[U^{i \hat{i}}(i, \hat{i} ; 0)\right] .
\end{aligned}
$$

Students of Poole (1970) will find it natural to begin the analysis of the effects of a 
velocity disturbance with the case in which hat chooses $\hat{i} \cdot{ }^{23}$ If hat chooses $\hat{i}$, nohat is better of choosing $i$ for all values of the parameters. Recall that the maximized value of nohat's zero-disturbance utility depends only on which instrument is chosen by hat and not on which instrument is chosen by nohat as stated in equations (58). Equations (64) and (58) together imply that

$$
\max _{i}\left[E U^{i \hat{i}}(i, \hat{i} ; v)\right]-\max _{m}\left[E U^{m \hat{i}}(m, \hat{i} ; v)\right]=.5\left[\alpha+\beta \rho\left(\frac{2 \phi}{\theta+\phi}\right)\right]^{2} \sigma_{v}^{2}>0 .
$$

The only way that both $i$ and $\hat{i}$ can be kept constant is if nohat offsets the effect of the velocity disturbance on the nohat money market and hat offsets the effect of the velocity disturbance on the hat money market. Thus, if nohat chooses $i$, a velocity disturbance has no effect on either target variable. However, if nohat chooses $m$, a positive velocity disturbance has the same effect as an increase in $m$ of equal size. With $\hat{i}$ fixed, an increase in $m$ increases $n$ and $\pi$.

Unfortunately, when hat chooses $\hat{m}$, the results are not so clear cut. If hat chooses $\hat{m}$, nohat is better off choosing $i$ if and only if that is, if and only if If nohat chooses $m$, the effects of an increase in $v$ are the same as the effects of an increase

\footnotetext{
${ }^{23}$ Turnovsky and d'Orey (1989) analyze instrument selection in a two-country model and obtain results similar to the ones reported in this Section.
} 


$$
\begin{aligned}
& \max _{i}\left[E U^{i \hat{m}}(i, \hat{m} ; v)\right]-\max _{m}\left[E U^{m \hat{m}}(m, \hat{m} ; v)\right]= \\
& -.5\left\{\left[\alpha-(\alpha+\beta \rho)\left(\frac{2 \phi}{\theta+\phi}\right)\right]^{2}-\alpha^{2}\right\} \sigma_{v}^{2}= \\
& .5\left[\alpha-(\alpha+\beta \rho)\left(\frac{2 \phi}{\theta+\phi}\right)+\alpha\right]\left[(\alpha+\beta \rho)\left(\frac{2 \phi}{\theta+\phi}\right)\right] \sigma_{v}^{2}>0, \\
& \alpha-(\alpha+\beta \rho)\left(\frac{2 \phi}{\theta+\phi}\right)>-\alpha .
\end{aligned}
$$

in $m$ and $\hat{m}$ by equal amounts. The increase in $\pi$ is

$$
\pi_{v}^{m \hat{m}}=\alpha,
$$

from equations (45). If nohat chooses $i$, the change in $\pi$ is

$$
\pi_{v}^{i \hat{m}}=\alpha-(\alpha+\beta \rho)\left(\frac{2 \phi}{\theta+\phi}\right) \gtreqless 0,
$$

by analogy with equations (55). The total effect of an increase in $v$ on $\pi$ in an im regime may be positive or negative and can be written as the sum of two effects: (1) the increase in $\pi$ resulting from an increase in $v$ under an $m \hat{m}$ regime which is equal to $\alpha$ and (2) the decrease in $\pi$ caused by the decrease in $m$ required to keep $i$ constant which is equal to $-(\alpha+\beta \rho)\left(\frac{2 \phi}{\theta+\phi}\right)$. The second effect is the product of the decrease in $m$ required to keep $i$ constant which is equal to $-\frac{2 \phi}{\theta+\phi}$ from equations (49) and the change in $\pi$ 
caused by a change in $m$ which is equal to $\alpha+\beta \rho$ from equations (45). The interpretation of the condition in equation (67) is that if hat chooses $\hat{m}$, nohat is better off choosing $i$ if and only if the change in $\pi$ when it chooses $i$ is greater in algebraic value than the negative of the change in $\pi$ when it chooses $m$.

Hat and nohat are symmetric. Therefore, if nohat chooses $i$, hat is better off choosing $\hat{i}$ for all parameter values. However, if nohat chooses $m$, hat is better ciff choosing $\hat{i}$ if and only if the condition in equation (67) is fulfilled. It follows that there is a unique equilibrium with $i$ and $\hat{i}$ chosen as instruments if and only if the condition in equation (67) is fulfilled. However, if this condition is not fulfilled there are two equilibria, one with $i$ and $\hat{i}$ chosen as instruments and another with $m$ and $\hat{m}$ chosen as instruments.

It is interesting that the condition in equation (67) is independent of the parameter $\delta$, the parameter which determines the amount by which the demand for nohat's good rises and the demand for hat's good falls when nohat's currency depreciates in real terms. Multiplying through the condition in equation (67) by $\theta+\phi$, rearranging, and using the definilion of $\theta$ given in equation (48) above yield

$$
2(\alpha \theta-\beta \rho \phi)=2 \rho\left[\alpha\left(\frac{1-2 \beta}{2}\right)-\beta \phi\right]>0
$$

where $\rho$ and $\theta$ are the only parameters that depend on $\delta$.

\section{Conclusions}


If there is no uncertainty and players can choose among instruments, there are multiple (Nash) equilibria in static, two-person, noncooperative, positive-sum games with linear reaztion functions. In this paper we confirm this assertion by providing a microeconomic example in which each duopolist can choose either price or quantity as an instrument and a macroeconomic example in which each of two countries can choose either a money supply or an interest rate as an instrument. Once one player has chosen his instrument and set a value for it, the other is indifferent between instruments. Therefore, there are four equilibria, one for each possible instrument pair.

In the microeconomic example, the equilibrium payoff of each player depends on the instruments chosen by both. However, in the microeconomic example, the equilibrium payoff of each player depends only on the instrument chosen by the other because of the behavior of wage setters.

We proceed to introduce uncertainty into both of our examples in the form of a play by nature represented by an additive disturbance. Introducing uncertainty in this form is of interest in its own right. However, we introduced it because we hoped that doing so would yield uniçue equilibria in our two examples. Our hope was based on Klemperer and Meyer (1986). They introduce an additive disturbance into the linear demand curves in a differentiated duopoly game in which the duopolists face independent, quadratic costs and find that the number of equilibria is reduced from four to one. The number of equilibria is reduced tecause with uncertainty even when one player has chosen his instrument and set a value for it, the other is not indifferent between instruments.

In our examples, introducing uncertainty reduces the number of equilibria, but 
sometimes to two instead of one. Furthermore, in both the Klemperer and Meyer example and in our examples with unique equilibria, for some parameter values with the smallest amount of uncertainty the symmetric instrument pair chosen in the unique equilibrium is the one that yields the lower payoff with no uncertainty. Further analysis of the implications of introducing uncertainty into deterministic games for the number of equilibria and for the characteristics of these equilibria seems to be in order. 


\section{References}

Barro, Robert, and David Gordon, (1983), "Rules, Discretion and Reputation in a Model of Monetary Policy," Journal of Monetary Economics, Vol. 12, No. 1, July, pF. 101-121.

Bertrand, Joseph, (1883), "Revue de la Theorie Mathematique de la Richesse Sociale et des Recherches sur les Principes Mathematiques de la Theorie des Richesses, "Journal des Savants, pp. 499-508.

Canzoneri, Matthew, and Dale Henderson, (1988), "Is Sovereign Policymaking Bad?," Carnegie-Rochester Series on Public Policy, Vol. 28, 1988, pp. 93-140.

Canzoneri, Matthew, and Dale Henderson, (1991), Monetary Policy in Interdependent Es:onomies: A Game Theoretic Approach, MIT Press, Cambridge.

Canzoneri, Matthew, and Dale Henderson, (1989), "Optimal Choice of Monetary Policy Instruments in a Simple Two-Country Game," in Frederick van der Ploeg and Aart de Zeeuw, eds., Dynamic Policy Games in Economics, North-Holland Publishing Company, Amsterdam.

Cheng, Leonard, (1985), "Comparing Bertrand and Cournot Equilibria: A Geometric Approach," Rand Journal of Economics, Vol. 16, No. 1, Spring, pp. 146-152.

Cournot, Augustin, (1838), Recherches sur les Principes Mathematiques de la Theorie des Richesses, L. Hachette, Paris.

Cooper, Russell, and Andrew John, (1988), "Coordinating Coordination Failures in Ke:ynesian Models," Quarterly Journal of Economics, vol. 100, no. 3, August, pp. 441-63.

Eijffinger, Sylvester, and Harry Huizinga, eds., (1996), Positive Political Economy: Theory and Evidence, Cambridge University Press, Cambridge.

Giavazzi, Francesco, and Alberto Giovannini, (1989), "Monetary Policy Interactions Under Managed Exchange Rates," Economica, Vol. 56, No. 222, May, pp. 199-213.

Henderson, Dale, and Ning Zhu, (1990), "Uncertainty and the Choice of Instruments in a Two-Country Policy Game," Open Economies Review, Vol. 1, No. 1, pp. 39-65.

Henderson, Dale, and Ning Zhu, (forthcoming), "Price and Quantity Competition in Differentiated Duopoly: How Cost Assumptions Matter," mimeo. 
Klemperer, Paul, and Margaret Meyer, (1986), "Price Competition vs. Quantity Competition: the Role of Uncertainty," Rand Journal of Economics, Vol. 17, No. 4, Winter, pp. 618-638.

Kydland, Finn, and Edward Prescott, (1977), "Rules Rather than Discretion: The Inconsistency of Optimal Plans," Journal of Political Economy, Vol. 85, Nc. 3, June, pp. 473-491.

Poole, William, (1970), "Optimal Choice of Monetary Policy Instruments in a Simple Stochastic Macro Model," Quarterly Journal of Economics, Vol. 84, No. 2. May, pp. 197-216.

Singh, Nirvikar, and Xavier Vives, (1984), "Price and Quantity Competition in a Differentiated Duopoly," Rand Journal of Economics, Vol. 15, No. 4, Winter, pp. 546-54.

Tabellini, Guido, (1987), "Optimal Monetary Instruments and Policy Games," Ricf:rche Economiche, Vol. 41, No. 3-4, July-December, pp. 315-325.

Turnovsky, Stephen, and Vasco d'Orey, (1986), "Monetary Policies in Interdependent Economies with Stochastic Disturbances: A Strategic Approach," Economir: Journal, Vol. 96, No. 383, September, 1986, pp. 696-721.

Turnovsky, Stephen, and Vasco d'Orey, (1989), "The Choice of Monetary Instrument in Two Interdependent Economies Under Uncertainty," Journal of Monetary Economics, Vol. 23, No. 1, January, pp. 121-133.

Vives, Xavier, (1985), "On the Efficiency of Bertrand and Cournot Equilibria with Product Differentiation," Journal of Economic Theory, Vol. 36, No. 1, June, pp. 166-175.

Weitzman, Martin, (1974), "Prices vs. Quantities," Review of Economic Studies, vol. 41, No. 4, October, pp. 477-491.

Xiang, Pinggui, (1993), Strategic Delegation, Ph. D. dissertation, University of Iowa.

Yohe, Gary, (1979), A Comparison of Price Controls and Quantity Controls, Garland Publishing, Inc., New York. 


\section{Table 1}

$\Pi^{(\hat{Q}}(Q, \hat{Q} ; \eta)=(a-g+\eta) Q-.5(b+k) Q^{2}-(f+u) \hat{Q} Q$

$$
\begin{aligned}
\left(\frac{b^{2}}{2}\right) \Pi^{P \hat{2}}(P, \hat{Q} ; \eta)= & {[b(a+g)+2 a k+(b+2 k) \eta] P-(b+k) P^{2} } \\
& +[b f g+(2 f k-b u)(a+\eta)] \hat{Q} \\
& -f(f k-b u) \hat{Q}^{2}-[f(b+2 k)-b u] P \hat{Q} \\
& -a(b g+a k)-(b g+2 a k) \eta-k \eta^{2},
\end{aligned}
$$

$$
\begin{aligned}
\left(2 b^{2}\right) \Pi^{(\hat{P}}(Q, \hat{P} ; \eta)= & {\left[2 a b(b-2 f)-2 b(b g+2 a u)-2\left(2 b f-b^{2}+2 b u\right) \eta\right] Q } \\
& \left.-b\left(b^{2}-4 f^{2}+b k-4 f u\right)\right] Q^{2}+4 b(f+u) \hat{P} Q,
\end{aligned}
$$

$$
\begin{aligned}
\left(\frac{\Delta^{2}}{2}\right) \Pi^{P \hat{P}}(P, \hat{P} ; \eta)= & \{\Delta b g+[\Delta+2 k b+2 u(b-2 f)](b-2 f)(a+\eta)\} P \\
& -\{2 f g \Delta+[4 f k-2 u(b-2 f)](b-2 f)(a+\eta)\} \hat{P} \\
& -b(\Delta+k b-4 u f) P^{2}-4 f(k f-b u) \hat{P}^{2} \\
& +2\left[f(\Delta+2 k b)-u\left(b^{2}+4 f^{2}\right)\right] P \hat{P} \\
& -g \Delta(b-2 f)(a+\eta)-(k+2 u)(b-2 f)^{2}(a+\eta)^{2}, \\
\Delta= & b^{2}-4 f^{2} .
\end{aligned}
$$


Table 2

$$
\begin{aligned}
U^{m \hat{m}}(m, \hat{m} ; v)= & {[\Psi-w(\alpha+\beta \rho)](m+v-w)+w \beta \rho(\hat{m}+v-\hat{w}) } \\
& -.5(\alpha+\beta \rho)^{2}(m+v-w)^{2}-.5(\beta \rho)^{2}(\hat{m}+v-\hat{w})^{2} \\
& +\beta \rho(\alpha+\beta \rho)(m+v-w)(\hat{m}+v-\hat{w})-.5 w^{2},
\end{aligned}
$$

$$
\begin{aligned}
(\theta+\phi)^{2} U^{i \hat{m}}(i, \hat{m} ; v)= & -(\theta+\phi)[\Psi-w(\alpha+\beta \rho)]\left(i-\pi_{+1}\right) \\
& +(\theta+\phi)\{\Psi(\theta-\phi)-w[\alpha(\theta+\phi)-(\alpha+\beta \rho) 2 \phi]\}(\hat{m}+v-\hat{w}) \\
& -.5(\alpha+\beta \rho)^{2}\left(i-\pi_{+1}\right)^{2} \\
& -.5[\alpha(\theta+\phi)-(\alpha+\beta \rho) 2 \phi]^{2}(\hat{m}+v-\hat{w})^{2} \\
& +(\alpha+\beta \rho)[\alpha(\theta+\phi)-(\alpha+\beta \rho) 2 \phi]\left(i-\pi_{+1}\right)(\hat{m}+v-\hat{w}) \\
& -.5(\theta+\phi)^{2} w^{2}
\end{aligned}
$$

$$
\begin{aligned}
(\theta+\phi)^{2} U^{m \hat{i}}(m, \hat{i} ; v)= & (\theta+\phi)\{(\theta+\phi) \Psi-w[\alpha(\theta+\phi)+\beta \rho(2 \phi)]\}(m+v-w) \\
& -w(\theta+\phi) \beta \rho\left(\hat{i}-\hat{\pi}_{+1}\right)-.5[\alpha(\theta+\phi)+\beta \rho(2 \phi)]^{2}(m+v-w)^{2} \\
& -.5(\beta \rho)^{2}\left(\hat{i}-\hat{\pi}_{+1}\right)^{2} \\
& -\beta \rho[\alpha(\theta+\phi)+\beta \rho(2 \phi)](m+v-w)\left(\hat{i}-\hat{\pi}_{+1}\right)-.5(\theta+\phi)^{2} \hat{w}^{2},
\end{aligned}
$$

$$
\begin{aligned}
16 \theta^{2} \phi^{2} U^{i \hat{i}}(i, \hat{i} ; v)= & -4 \theta \phi\left[\Psi(\theta+\phi)-w[\alpha(\theta+\phi)+\beta \rho(2 \phi)]\left(i-\pi_{+1}\right)\right. \\
& -4 \theta \phi\left[\Psi(\theta-\phi)-w[\alpha(\theta+\phi)-(\alpha+\beta \rho) 2 \phi]\left(\hat{i}-\hat{\pi}_{+1}\right)\right. \\
& -.5[\alpha(\theta+\phi)+\beta \rho(2 \phi)]^{2}\left(i-\pi_{+1}\right)^{2} \\
& -.5[\alpha(\theta+\phi)-(\alpha+\beta \rho) 2 \phi]^{2}\left(\hat{i}-\hat{\pi}_{+1}\right)^{2} \\
& +[\alpha(\theta+\phi)+\beta \rho(2 \phi)] \\
& \times[\alpha(\theta+\phi)-(\alpha+\beta \rho) 2 \phi]\left(i-\pi_{+1}\right)\left(\hat{i}-\hat{\pi}_{+1}\right)-8 \theta^{2} \phi^{2} w^{2} .
\end{aligned}
$$




\section{International Finance Discussion Papers}

IFDP

Number
Titles

1995

526 Uncertainty, Instrument Choice, and the Uniqueness of Nash Equilibrium: Microeconomic and Macroeconomic Examples

525 Targeting Inflation in the 1990s: Recent Challenges

524 Economic Development and Intergenerational Economic Mobility

$523 \quad$ Human Capital Accumulation, Fertility and Growth: A Re-Analysis

522 Excess Returns and Risk at the Long End of the Treasury Market: An EGARCH-M Approach

521 The Money Transmission Mechanism in Mexico

520 When is Monetary Policy Effective?

519 Central Bank Independence, Inflation and Growth in Transition Economies

518 Alternative Approaches to Real Exchange Rates and Real Interest Rates: Three Up and Three Down

517 Product market competition and the impact of price undertainty on investment: some evidence from U.S. manufacturing industries

$516 \quad$ Block Distributed Methods for Solving Multi-country Econometric Models

515 Supply-side sources of inflation: evidence frp, OECD countries

514 Capital Flight from the Countries in Transition: Some Theory and Empirical Evidence
Author(s)

Dale W. Henderson

Ning S. Zhu

Richard T. Freeman Jonathan L. Willis

Murat F. Iyigun

Murat F. Iyigun
Allan D. Brunner
David P. Simon
Martina Copelman
Alejandro M. Werner
John Ammer
Allan D. Brunner
Prakash Loungani
Nathan Sheets

Hali J. Edison

William R. Melick

Vivek Ghosal

Prakash Loungani

Jon Faust

Ralph Tryon

Prakash Loungani

Phillip Swagel

Nathan Sheets

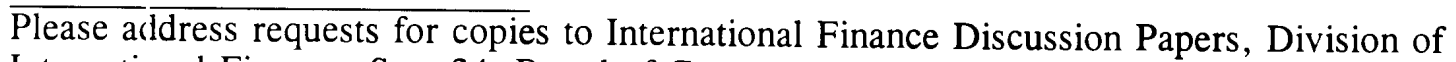
International Finance, Stop 24, Board of Governors of the Federal Reserve System, Washington, D.C. 20551. 


\section{International Finance Discussion Papers}

IFDP

Number

513 Bank Lending and Economic Activity in Japan: Did "Financial Factors" Contribute to the Recent Downturn?

Titles

1995 of U.S. Manufacturing Industries Superneutrality Conjecture

Import Prices and the Competing Goods Effect

Supply-side Economics in a Global Economy

The Lucas Critique In Practice: Theory Without Measurement Instability

Inferences from Parametric and Non-Parametric Covariance Matrix Estimation Procedures Initial Real Effects of Credible Plans

Strategic Returns to International

Diversification: An Application to the Equity

Markets of Europe, Japan, and North America

Real Exchange Rate Movements in High Inflation Countries

Political Competition, Casual Relations Between
Author(s)

\section{Evidence on Nominal Wage Rigidity From a Panel}

Do Taxes Matter for Long-Run Growth?: Harberger's

Options, Sunspots, and the Creation of Uncertainty

Hysteresis in a Simple Model of Currency Substitution

Real Exchange Rate Targeting and Macroeconomic

Exchange-Rate Based Inflation Stabilization: The Taxes and Spending, and Their Influence on Government Size: Evidence From State-Level Data
Allan D. Brunner

Steven B. Kamin

Vivek Ghosil

Prakash Loungani

Enrique G. Mendoza

Gian Maria Milesi-Ferretti

Patrick Aseč

David Bowman

Jon Faust

Martin Urib:

Phillip Swagel

Enrique G. Mendoza

Linda L. Tesar

Neil R. Ericsson

John S. Irons

Martin Urib!:

Wouter J. Den Haan

Andrew T. l.evin

Martin Urib:

John Ammer

Jianping $\mathrm{Me}$

John H. Rogers

Ping Wang

Diane Lim Fogers

John H. Rogers 